\title{
On-State Voltage Measurement of Fast Switching Power Semiconductors
}

\author{
Mattia Guacci, Dominik Bortis, and Johann W. Kolar
}

\begin{abstract}
The on-state resistance $\boldsymbol{R}_{\mathrm{ds}, \mathrm{on}}$ is a key characteristic of unipolar power semiconductors and its value depends on the operating conditions, e.g. junction temperature, conducted current and applied gate voltage. Hence, the exact determination of the $\boldsymbol{R}_{\mathrm{ds}, \text { on }}$ value cannot rely on datasheet information and requires the measurement of current and on-state voltage during operation. Besides the determination of the conduction losses, the onstate voltage measurement enables dynamic $\boldsymbol{R}_{\mathrm{ds}, \text { on }}$ analysis, device temperature estimation, condition monitoring and consequently time-to-failure prediction. However, in contrast to a switch current measurement, several challenges arise in the design of an on-state voltage measurement circuit (OVMC), i.e. high measurement accuracy ( $\mathrm{mV}$-range) during on-state, high blocking voltage capability (kV-range) during off-state and fast dynamic response (ns-range) during switching transitions are demanded. Different OVMC concepts are known from IGBT applications, however, the more severe requirements introduced from the high switching frequency and low $\mathrm{OV}$ characterizing the operation of fast switching power semiconductors, prevent their usage. Off-the-shelf products hardly satisfy the mentioned specifications, whereas the performance of state-of-the-art OVM research prototypes require further investigations and/or improvements. With this aim, an innovative OVMC concept is designed, analyzed, calibrated and tested in this paper. Furthermore, the conduction losses of different power semiconductors are measured as function of their operating conditions to validate the performance and highlight the potential of the proposed OVMC.
\end{abstract}

Index Terms - Conduction loss measurement, on-state resistance, on-state voltage measurement circuit.

\section{INTRODUCTION}

$\mathrm{T}$ ODAY, the detailed experimental analysis of a power converter circuit is typically focused on the switching behavior of the power semiconductors, e.g. switching overvoltages and voltage oscillations occurring in hard-switching [1] and/or switching voltage and current time displacement relative to zero-voltage switching (ZVS) [2]. However, with the increasing switching speed enabled by wide bandgap devices and/or with employing ZVS concepts, the loss contribution of the semiconductors is more and more dominated by the conduction losses.

Manuscript received February 1, 2018. This work was supported by the Swiss State Secretariat for Education, Research and Innovation (SERI) under contract number 15.0161. The opinions expressed and arguments employed herein do not necessarily reflect the official views of the Swiss Government. (Corresponding author: Mattia Guacci.)

The authors are with Power Electronic Systems Laboratory, ETH Zurich, Switzerland (e-mail: guacci@lem.ee.ethz.ch).

Digital Object Identifier 10.24295/CPSSTPEA.2018.00016
The power converter operating conditions are significantly influencing the occurring conduction losses. Junction temperature, conducted current, applied gate voltage, switching frequency, manufacturing variability and aging take, in fact, a combined impact on the instantaneous value of the semiconductors on-state resistance $R_{\mathrm{d}, o n}$. Despite this premise and contrary to switching loss analysis, no on-state behavior analysis and/ or no experimental verification of the calculated $R_{\mathrm{ds} ; \text { on }}$ value is typically carried. Hence, worst-case approximations, based on datasheet information, are inferred to estimate the conduction losses. Performing online $R_{\mathrm{ds}, \text { on }}$ measurements would allow to accurately verify conduction loss calculations and hence to improve optimization procedures for the design of power converters [3], [4]. In order to achieve this, the voltage across and the current through the device-under-test (DUT) have to be measured accurately and simultaneously. Several solutions are suitable to obtain the DUT current, i.e. it can be directly measured (e.g. with a series connected current shunt) or indirectly derived from the load current and the gate signals, both available for control purposes. In contrast, the usage of conventional voltage probes to accurately measure the DUT on-state voltage $(\mathrm{OV})$ is prevented from the resulting dynamic range, i.e. the ratio between the maximum voltage (during off-state) and the minimum voltage (during on-state) applied at the input of the measurement circuit. The extreme required dynamic range limits the measurement resolution. This can be understood considering an example. If a conventional 12-bit oscilloscope is set to capture the voltage blocked (e.g. $1000 \mathrm{~V})$ from a specimen power MOSFET (e.g. $R_{\mathrm{ds}, \mathrm{on}}=25 \mathrm{~m} \Omega$ ), the least significant bit, i.e. the measurement resolution, corresponds to $1000 \mathrm{~V} / 2^{12} \approx$ $250 \mathrm{mV}$. This voltage is already half the $\mathrm{OV}$ of the mentioned MOSFET conducting $20 \mathrm{~A}(25 \mathrm{~m} \Omega \cdot 20 \mathrm{~A}=500 \mathrm{mV})$ and the resulting accuracy is definitely insufficient. Ideally, in order to perform this measurement with an accuracy of $1 \%$, the least significant bit should correspond to $5 \mathrm{mV}(0.01 \cdot 500 \mathrm{mV})$; in other words, an 18-bit oscilloscope $\left(1000 \mathrm{~V} / 2^{18} \approx 4 \mathrm{mV}\right)$ would be required [5]. Alternatively, the conventional oscilloscope fullscale voltage would have to be limited to $20 \mathrm{~V}\left(20 \mathrm{~V} / 2^{12} \approx 5\right.$ $\mathrm{mV}$ ). The full-scale voltage reduces to $1 \mathrm{~V}$ if the same accuracy is desired while measuring only $1 \mathrm{~A}(25 \mathrm{~m} \Omega \cdot 1 \mathrm{~A}=25 \mathrm{mV}$, $0.01 \cdot 25 \mathrm{mV}=0.25 \mathrm{mV}$ and $0.25 \mathrm{mV} \cdot 2^{12}=1 \mathrm{~V}$ ). However, if the oscilloscope range is limited to measure the sole OV (e.g. $1 \mathrm{~V}$ ), the overdrive of the oscilloscope amplifiers caused by the voltage blocked in off-state significantly distorts the measurements and could damage the instrument.

It results clear that the achievable accuracy is insufficient because of the trade-off with the excessive dynamic range. Therefore, only dedicated on-state voltage measurement cir- 
cuits (OVMCs), limiting the voltage during off-state to values within the oscilloscope input voltage range, allow accurate measurements during on-state. For example, a full-scale voltage of $10 \mathrm{~V}$ guarantees a measurement resolution of $10 \mathrm{~V} / 2^{12} \approx 2.5$ $\mathrm{mV}$, sufficient in most cases.

Beside accuracy and resolution, also the bandwidth of OVMCs is of importance, since modern Gallium-Nitride (GaN) semiconductors often present dynamic $R_{\mathrm{ds}, \text { on }}$ effects [6], [7], i.e. a high initial (i.e. immediately after a turn-on switching transition) $R_{\mathrm{ds}, \text { on }}$ value which slowly (i.e. with time constants varying in the $\mu$ s-range) settles to a constant value. In order to characterize this phenomenon, OVMCs should measure the correct OV immediately after the turn-on transition of the DUT. The dynamic $R_{\mathrm{ds}, o n}$ is a fundamental figure-of-merit in the evaluation of $\mathrm{GaN}$ semiconductors and it depends on the voltage blocked during off-state, as well as on the switching frequency [7]-[9]. Being this information not specified in the datasheet, the importance of the experimental on-state behavior analysis is remarked.

In addition to dynamic and stationary OV analysis, other application areas are envisaged in literature, resulting in requirements of an ideal OVMC. The OV is a promising temperature sensitive electrical parameter, i.e. a circuit parameter ensuring high sensitivity and good linearity with respect to temperature variations [10]. Measuring the OV offers the potential for replacing conventional temperature measurements in application with severe volume constraints. Furthermore, the $\mathrm{OV}$ is identified as a favorable aging indicator. E.g. in power modules, a crack in the metallization layer and/or the lift-off of a bond-wire cause an increase of the $R_{\mathrm{ds}, \text { on }}$ value, i.e. of the OV [11], [12]. This establishes a positive feedback mechanism accelerating the aging process. Monitoring the OV allows a detection of the failure and potentially time-tofailure predictions, advantageous e.g. in reliability critical or remote applications. Accordingly, a trend towards intelligent gate drivers integrating OVMCs is traced [13] and, to facilitate this, compactness is generally demanded. As well the mentioned bandwidth requirement, associated to a fast dynamic response of the OVMC after a switching transition, is of importance in all the foreseen application areas, if fast switching semiconductors are considered.

Finally, the online measurement of conduction losses enabled by OVMCs can significantly improve the measurement accuracy of calorimetric switching loss measurement methods [14]-[16]. This application is commented in detail in Section IV-C, since it constitutes a main reason for this research work. Summarizing, high accuracy, fast dynamic response, low complexity, and high compactness are the desirable characteristics of OVMCs.

The nowadays most common OVM approaches can be traced back more than thirty years [17], [18]. Recently, the increased interest of the power electronic community in wide bandgap semiconductors, combining reduced $R_{\mathrm{ds}, \text { on }}$ values with increased switching speeds, motivated the interest to derive solutions offering better accuracy and higher bandwidth, e.g. [19] and [20], where also a comprehensive

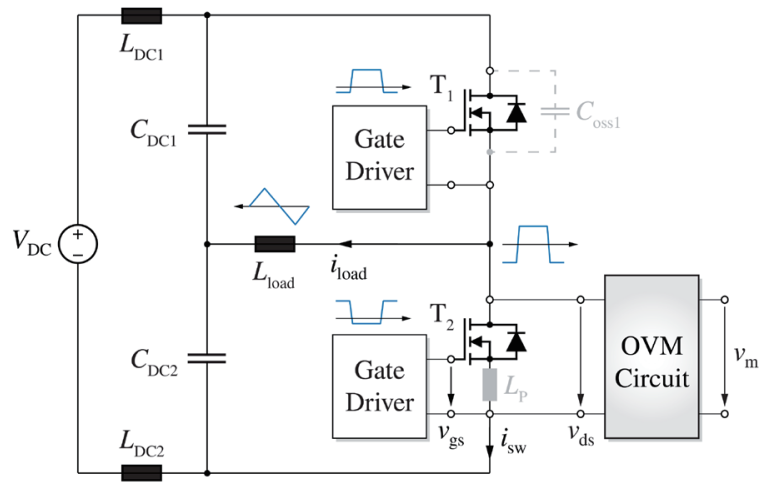

Fig. 1. Power converter setup considered as reference for the overall analysis. The two power semiconductors in half-bridge configuration $\mathrm{T}_{1}$ and $\mathrm{T}_{2}$ are operated with complementary $50 \%$ duty cycles. The output inductor $L_{\text {load }}$ tapped to the split DC link capacitors $C_{\mathrm{DC} 1}$ and $C_{\mathrm{DC} 2}$ ensures symmetric triangular current mode (TCM) operation (blue waveforms). The parasitic inductance $L_{\mathrm{P}}$ and parasitic output capacitance of $\mathrm{T}_{1}$ (i.e. $C_{\mathrm{oss}}$ ) are as well shown. An OVMC (gray) is connected in parallel to the low-side semiconductor $\mathrm{T}_{2}$, enabling online $R_{\mathrm{ds}, \mathrm{on}}$ measurements.

overview of the state-of-the-art is discussed. However, these concepts still reveal limited performance: [20] suffers significantly from noise and common-mode disturbances, and is tested only at high OV values, while [18] and [19] have an intrinsic accuracy limitation, i.e. an unknown diode forward voltage drop in the OVM path, as discussed in Section II. A solution to the latter issue is proposed in [12], where a novel OVMC is presented to monitor the wear-out of high power IGBT in power modules. Unfortunately, the performance of this OVMC highlighted in [12], [21], [22] and the provided guidelines are insufficient and inadequate for fast switching power semiconductors, especially in terms of bandwidth and dynamic response. Significant distortions are introduced from this OVMC at its output for tens of $\mu$ s and also the settling time of the designed analog circuitry itself is already in the range of $1 \mu$ s, i.e. comparable to a conduction period of a device operating with a switching frequency in the hundreds of kHz-range. Moreover, with respect to IGBTs, where the $\mathrm{OV}$ is rarely below $500 \mathrm{mV}$, when considering fast switching semiconductors with $R_{\mathrm{d}, \mathrm{on}}$ values in the $\mathrm{m} \Omega$ -range, it results significantly more challenging to guarantee the same OVM accuracy. Only limited information on the accuracy are provided in [12] and the performed OVMs are not completely validated with an alternative setup. Nevertheless, this OVMC is considered as a promising solution for the foreseen achievable accuracy enabled by the correction of the unknown diode forward voltage drop in the OVM path. Lately, also OVM probes became commercially available [23], [24], aiming to replace OVM integrated circuits [25]. A commercial solution is tested, but poor dynamic performance is experienced, as commented in Section III-D. Consequently, it results necessary to develop an OVMC able to satisfy all above-mentioned requirements in order to cover all applications.

The reference setup, supporting the analysis presented in this paper, is shown in Fig. 1. It highlights an OVMC (gray) 
connected to the low-side power semiconductor (i.e. $\mathrm{T}_{2}$, the DUT) of an half-bridge configuration. The same circuit could be connected to the high-side $\mathrm{T}_{1}$, however, for simplicity, only the depicted solution is analyzed in the following. Different power semiconductors are then selected for $\mathrm{T}_{2}$, enabling their comparison.

The proposed OVMC is presented in Section III, where the achieved accuracy and bandwidth are verified through measurements on a calibrated test-bench. Afterwards, the turnon behavior of different Silicon (Si), Silicon-Carbide $(\mathrm{SiC})$ and Gallium-Nitride $(\mathrm{GaN})$ power semiconductors is experimentally analyzed, validating the dynamic performance of this OVMC. The main challenges faced during the online measurement of conduction losses are commented in Section IV-A, anticipating the measurement results discussed in Section IV-B (conduction losses) and Section IV-C (switching losses). Conclusions are presented in Section V.

\section{Conventonal On-State Voltage Measurement CIRCUITS}

The schematics depicted in Fig. 2 show the two most common implementations of OVMCs. They ideally perform as a shortcircuit (i.e. $v_{\mathrm{ds}} \approx v_{1, \mathrm{a}}$ and $v_{1, \mathrm{~b}}$ ) when the power transistor $\mathrm{T}_{2}$ (i.e. DUT, see Fig. 1) is conducting (on-state) and as an open-circuit when it is blocking the DC-link voltage $V_{\mathrm{DC}}$ (offstate). As explained in the introduction, the accuracy of $v_{1}$ during on-state and the dynamic performance of the transition between the two states are the most important figures-of-merit of an OVMC. These two approaches are compared according to these criteria in the rest of this section. The final aim is to highlight their respective advantages in order to facilitate the design of a better performing solution.

The OVMC illustrated in Fig. 2(a) [18] is based on the MOSFET $T_{p}$. The gate of $T_{p}$ is connected to a voltage source $V_{\mathrm{p}}$ and when $\mathrm{T}_{\mathrm{p}}$ is in on-state, i.e. $V_{\mathrm{p}}>v_{\mathrm{l}, \mathrm{a}}+V_{\mathrm{th}, \mathrm{Tp}}$, the measured voltage

$$
v_{1, \mathrm{a}}=\frac{R_{1}}{R_{1}+R_{\mathrm{T}_{\mathrm{p}}}} v_{\mathrm{ds}}
$$

$V_{\mathrm{th}, \mathrm{Tp}}$ and $R_{\mathrm{Tp}}$ are the threshold voltage and the on-state resistance of $\mathrm{T}_{\mathrm{p}}$, respectively. Typically $R_{\mathrm{Tp}} \ll R_{1}$, therefore $v_{1, \mathrm{a}}$ $\approx v_{\mathrm{ds}}$. Regarding the transition between the two states, two control strategies are possible:

- active: $V_{\mathrm{p}}$ is switched to $0 \mathrm{~V}$ before $\mathrm{T}_{2}$ is turned off and back to $V_{\mathrm{p}}$ after $\mathrm{T}_{2}$ is completely turned on;

- passive: $V_{\mathrm{p}}$ is kept constant and $\mathrm{T}_{\mathrm{p}}$ operates in its linear region during the off-state of $\mathrm{T}_{2}$.

The active solution is intuitively effective but increases the complexity of this OVMC, e.g. logic and delay circuitry between the gate drivers of $T_{2}$ and $T_{p}$ results necessary and introduces a blanking time. Therefore, the less intuitive passive solution is preferred and herein considered, however additional challenges need to be faced [20].

The OVMC of Fig. 2 (b) [17] is realized with the diode $\mathrm{D}_{1}$ instead of the MOSFET $\mathrm{T}_{\mathrm{p}}$. When $v_{\mathrm{ds}}<V_{\mathrm{p}}-V_{\mathrm{fv}, \mathrm{D}}, \mathrm{D}_{1}$ con-

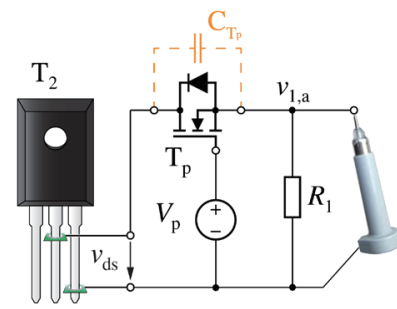

(a)

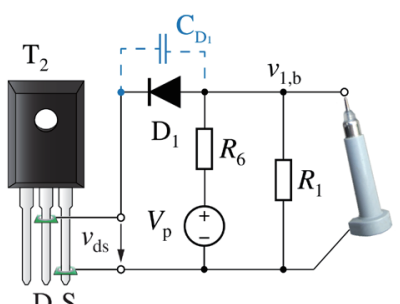

(b)
Fig. 2. Schematic representation of the two most common implementations of OVMCs. The input voltage $v_{\mathrm{ds}}$ is sensed at the DUT leads (see Fig. 1); by means of the MOSFET $\mathrm{T}_{\mathrm{p}}$ (a) or of the diode $\mathrm{D}_{1}(\mathrm{~b})$, the output is decoupled during the DUT off-state and connected during its on-state. While the dynamic performance of (a) are affected from $\mathrm{C}_{\mathrm{T}_{\mathrm{p}}}$, the accuracy of (b) is compromised by $v_{\mathrm{D} 1}$.

ducts and

$$
v_{1, \mathrm{~b}}=v_{\mathrm{ds}}+v_{\mathrm{D}_{1}}\left(T_{\mathrm{D}_{1}}, i_{\mathrm{D}_{1}}\right) .
$$

$V_{\mathrm{fv}, \mathrm{Dl}}, T_{\mathrm{Dl}}$ and $i_{\mathrm{D} 1}$ are the forward voltage, the junction temperature and the current of $\mathrm{D}_{1}$ respectively. During off-state, $v_{1, \mathrm{~b}}$ is fixed from $V_{\mathrm{p}}$ and $\mathrm{D}_{1}$ naturally blocks $V_{\mathrm{DC}}$. Differently from (1), a significant error term (i.e. $v_{\mathrm{D} 1}$ in the range of hundreds of $\mathrm{mV}$ ) appears in (2), causing an offset on $v_{1, \mathrm{~b}}$. As specified in (2), $v_{\mathrm{D} 1}$ is function of $T_{\mathrm{D} 1}$ and $i_{\mathrm{D} 1}$, however, an approximate value is commonly subtracted from $v_{1, b}$ to obtain $v_{\mathrm{ds}}$ [18], [19]. This only partially compensates it and compromises the accuracy of the measurement. Nevertheless, this OVMC is often preferred (e.g. in desaturation circuits) to the one previously described, giving lower importance to the achievable accuracy than to hardware complexity and dynamic performance. The latter aspect is the focus of the following paragraph.

The transition between the two DUT states, i.e. the commutation of the half-bridge (Fig. 1), implies charge and discharge of the parasitic output capacitances of $\mathrm{T}_{1}$ and $\mathrm{T}_{2}$ (i.e. $C_{\text {oss } 1}$ and $C_{\text {oss } 2}$ in Fig. 1) as well as of the input capacitance of the OVMCs. In fact, $C_{\mathrm{T}_{\mathrm{p}}}$ (orange in Fig. 2(a)) and $C_{\mathrm{D}_{1}}$ (blue in Fig. 2(b)) are charged to $V_{\mathrm{DC}}$ when $\mathrm{T}_{2}$ is in off-state while are discharged when $\mathrm{T}_{2}$ is conducting. The charging/ discharging network includes the resistor $R_{1}$ or $R_{6}$, whose range of suitable values is limited from the operation of the OVMCs (e.g. $R_{1}$ defines the off-state current in the MOSFET-based solution). Consequently, $C_{\mathrm{T}_{\mathrm{p}}}$ and $C_{\mathrm{D}_{1}}$ should be minimized:

- compared to $C_{\text {oss }}$ in order not to affect the switching transition of the half-bridge, i.e. slowing down the voltage slope of the switching node $\mathrm{d} v_{\mathrm{d}} / \mathrm{d} t$ in soft-switching transitions or significantly increasing the capacitive energy dissipated in hard-switching transitions;

- accordingly minimizing the magnitude of the charging/ discharging current spikes, potentially damaging measurement and/or supply circuitry;

- minimizing the time constant of the $R C$-network formed with $R_{1}$ or $R_{6}$, improving the dynamic performance of the OVMCs (cf. Section III-D). 


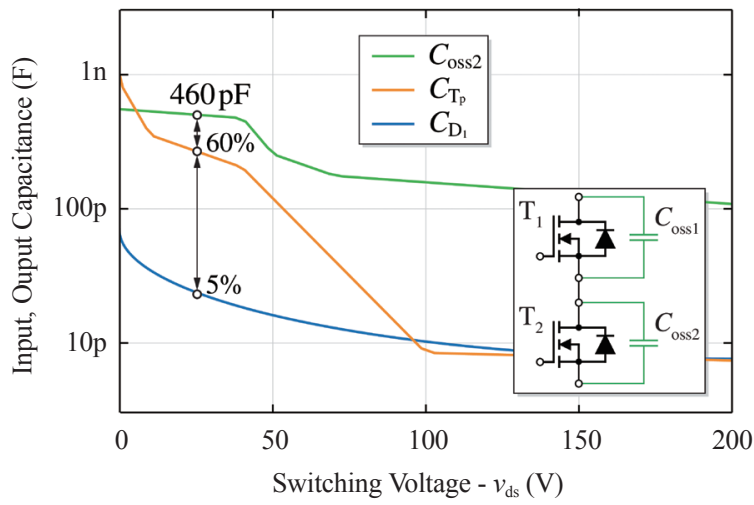

(a)

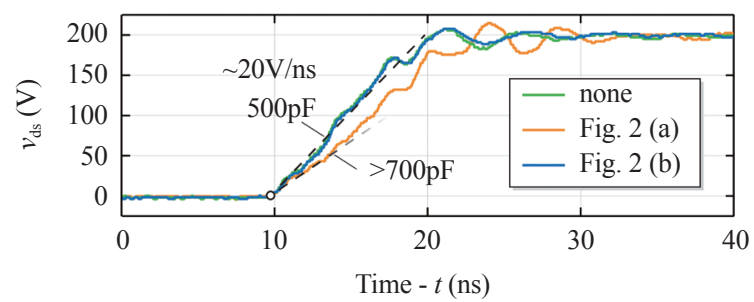

(b)

Fig. 3. (a) Comparison of the parasitic capacitances of the devices selected to realize the OVMCs of Fig. 2 . For $v_{\mathrm{ds}}<100 \mathrm{~V}, C_{\mathrm{D}_{1}}$ is considerably smaller than $C_{\mathrm{T}_{\mathrm{p}}}$ and, more important, negligible compared to $C_{\mathrm{oss} 2}$. This reflects on the switching behavior of the half-bridge (b): the presence of $\mathrm{T}_{\mathrm{p}}$, i.e. $C_{\mathrm{T}_{\mathrm{p}}}$ (orange), significantly reduces $\mathrm{d} v_{\mathrm{ds}} / \mathrm{d} t$ during a soft-switching turn-off transition of $\mathrm{T}_{2}$, whereas $C_{\mathrm{D}_{1}}$ (blue) does not have any influence.

These considerations clearly address the selection of $T_{p}$ and $\mathrm{D}_{1}$ towards devices featuring a small parasitic capacitance. Additionally, a low-inductance package and a short connection from the OVMC to the DUT are preferred in order to limit the voltage oscillations of $v_{1}$ inevitably excited from the switching transitions. According to these criteria, in the considered setups, a $800 \mathrm{~V} \mathrm{~N}$-channel Si MOSFET [26] is selected for $\mathrm{T}_{\mathrm{p}}$ as in [10] and a $600 \mathrm{~V} \mathrm{SiC}$ Schottky diode [27] for $\mathrm{D}_{1}$.

In order to prove the first statement, the parasitic capacitances of the selected devices are compared with the parasitic output capacitance $C_{\text {oss } 2}$ of a commercial $650 \mathrm{~V}$ E-mode GaN HEMT (Specimen C in TABLE II) as function of $v_{\mathrm{ds}}$ in Fig. 3(a). At $v_{\mathrm{ds}}=25 \mathrm{~V}, C_{\mathrm{D}_{1}}$ is a negligible fraction (i.e. $5 \%$ ) of $C_{\text {oss2 } 2}$, while $C_{\mathrm{T}_{\mathrm{p}}}$ contributes to the overall capacitance (i.e. $C_{\text {oss } 2}+C_{\mathrm{T}_{\mathrm{p}}}$ ) for more than $30 \%$. As mentioned, this influences the switching behavior of the half-bridge where Specimen $\mathrm{C}$ is selected for $\mathrm{T}_{2}$, e.g. in soft-switching operation. In Fig. 3 (b), measured waveforms of $v_{\mathrm{ds}}$ and $\mathrm{d} v_{\mathrm{ds}} / \mathrm{d} t$ for three different conditions are shown: without any OVMC (green), with the MOSFET-based approach (orange) and with the diode-based approach (blue) connected to $\mathrm{T}_{2}$. While the presence of $\mathrm{D}_{1}$ does not affect $\mathrm{d} v_{\mathrm{ds}} / \mathrm{d} t \approx 20 \frac{\mathrm{V}}{\mathrm{ns}}, \mathrm{T}_{\mathrm{p}}$ slows it down especially in the first phase where $C_{\mathrm{T}_{\mathrm{p}}}$ is comparable with $C_{\mathrm{oss} 2}$. This effect is clearly undesired and can be attributed to the selected $T_{p}$. However, depending on $T_{2}$, the range of suitable $T_{p}$ (in terms of $C_{\mathrm{T}_{\mathrm{p}}}$ ) narrows, reaching a bottleneck in the case of interest of fast switching power semiconductors. Differently, par- asitic capacitance of $600 \mathrm{~V}$ commercially available diodes can be in the range of few $\mathrm{pF}$, enabling a more general OVM solution. Consequently, the approach presented in the next section is derived from the diode-based circuit. It aims to improve its accuracy, while benefiting from the reduced parasitic capacitance.

\section{Proposed On-State Voltage Measurement Circuit}

In Section II, strengths and weaknesses of the two most common OVM approaches are highlighted. In this section, a promising solution derived from the diode-based circuit and originally presented in [12], is analyzed in detail. As discussed in the introduction, it results significantly more challenging to perform OVMs in the case of fast-switching semiconductors compared to IGBTs, e.g. due to the higher switching frequency and lower OV. Considering the design guidelines proposed herein, relative in particular to the small input parasitic capacitance, the usage of a $50 \Omega$ output stage, the high-bandwidth conditioning circuitry, the integrated generation of the supply voltages and the thoughtful design and calibration process, this OVMC is improved to combine high accuracy, outstanding dynamic performance and reduced circuit complexity. This ultimately enables its usage in the mentioned application areas of interest, as verified with the described measurement results. Initially, the operating principle of this OVMC is presented to better comprehend its possible limitations, providing the basis for its improvement.

\section{A. Operating Principle}

The schematic of the proposed OVMC for fast switching power semiconductors is depicted in Fig. 4(a) (a simplified version is in Fig. 4(c)) together with its hardware realization (Fig. 4(b)). The concept is derived from the approach shown in Fig. 2(b), but now two identical diodes (i.e. $\mathrm{D}_{1}$ and $\mathrm{D}_{2}$ ) are connected in series at the input. During the on-state of $\mathrm{T}_{2}$ (see Fig. 2(c)), the same current $i_{\mathrm{D}}$ is assumed to flow through $\mathrm{D}_{1}$ and $\mathrm{D}_{2}\left(i_{\mathrm{D}_{1}}=i_{\mathrm{D}_{2}}\right)$, therefore, given the same operating point for the two diodes, the respective voltage drops $v_{\mathrm{D}}$ are assumed to be identical $\left(v_{\mathrm{D}_{1}}=v_{\mathrm{D}_{2}}\right)$. Since thanks to the zener diode $\mathrm{Z}_{1}$, only $\mathrm{D}_{1}$ blocks $V_{\mathrm{DC}}, v_{\mathrm{D}_{2}}$ can be measured and subtracted (with the operational amplifier $\mathrm{Op}_{2}$ ) from $v_{1}$. Consequently, the offset $v_{D_{1}}$ present in (2) can be, with this OVMC, exactly corrected rather than roughly compensated. During off-state, the operation is similar to the one of the OVMC in Fig. 2(b).

The transfer function of the complete OVMC (Fig. 4(a)) is herein derived. In the case of $v_{\mathrm{ds}}<V_{\mathrm{p}}-v_{\mathrm{D}_{2}}-v_{\mathrm{D}_{1}}$ and with $\mathrm{Op}$ and $\mathrm{Op}_{2}$ operating linearly (always assumed from here on),

$$
\left\{\begin{array}{l}
v_{+}=\frac{R_{1 \mathrm{~b}}}{R_{1 \mathrm{a}}+R_{1 \mathrm{~b}}} v_{1} \\
v_{3}=\frac{R_{2 \mathrm{~b}}}{R_{2 \mathrm{a}}+R_{2 \mathrm{~b}}} v_{2} \\
v_{\mathrm{m}}=v_{-}-\frac{R_{4}}{R_{3}}\left(v_{3}-v_{-}\right)
\end{array}\right.
$$




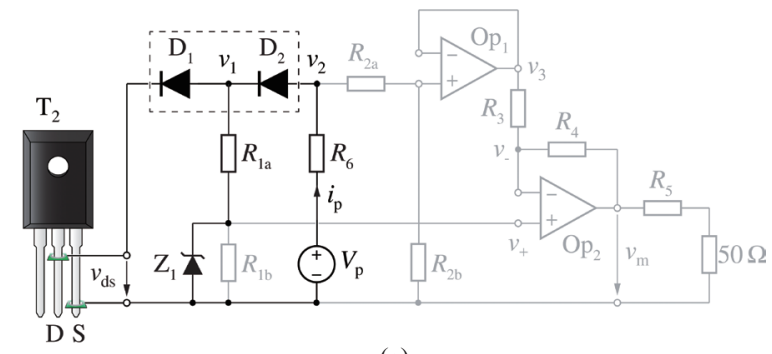

(a)

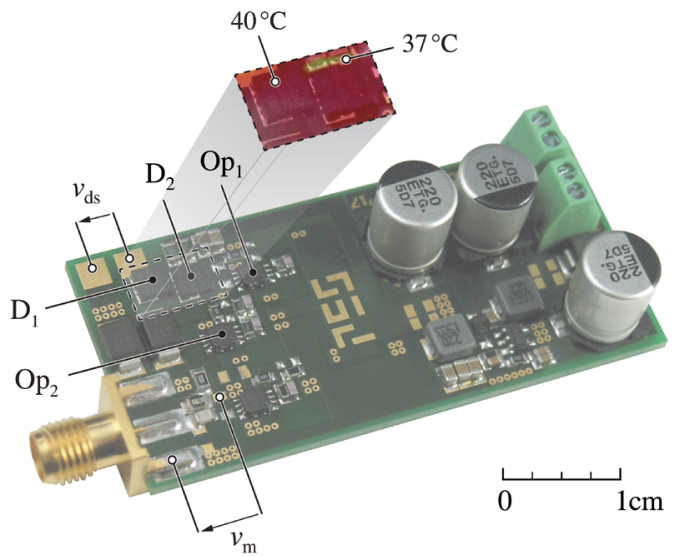

(b)

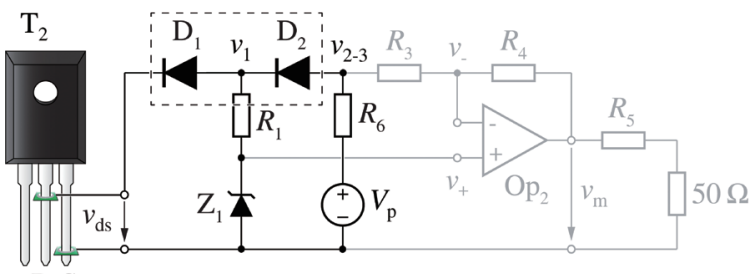

(c)

Fig. 4. Schematic representation of the realized (a) and simplified (c) versions of the proposed OVMC. (b) depicts the hardware realization of (a). $\mathrm{D}_{1}$ and $\mathrm{D}_{2}$ are thermally well coupled (dashed box) and electrically connected in series at the input of the OVMC in order to improve the measurement accuracy.

holds. The system of equations given in (3) can be solved for the output voltage $v_{\mathrm{m}}$ if $v_{+}=v_{-}$is assumed, obtaining

$$
v_{\mathrm{m}} \stackrel{(3)}{=} \frac{R_{1 \mathrm{~b}}}{R_{1 \mathrm{a}}+R_{1 \mathrm{~b}}}\left(1+\frac{R_{4}}{R_{3}}\right) v_{1}-\frac{R_{2 \mathrm{~b}}}{R_{2 \mathrm{a}}+R_{2 \mathrm{~b}}} \frac{R_{4}}{R_{3}} v_{2}
$$

Defining $R_{1, \mathrm{~b}}=R_{2, \mathrm{~b}} \doteq R_{\beta}$ and $R_{1, \mathrm{a}}=R_{2, \mathrm{a}} \doteq \beta R_{\beta}$ so that

$$
\frac{R_{1 \mathrm{~b}}}{R_{1 \mathrm{a}}+R_{1 \mathrm{~b}}}=\frac{R_{2 \mathrm{~b}}}{R_{2 \mathrm{a}}+R_{2 \mathrm{~b}}}=\frac{1}{1+\beta}
$$

and selecting $R_{3}=R_{4}$, (4) can be simplified to

$$
v_{\mathrm{m}} \stackrel{(4)}{=} \frac{1}{1+\beta}\left(2 v_{1}-v_{2}\right) \text {. }
$$

Finally, it must be noticed that the term

$$
2 v_{1}-v_{2}=v_{1}-\left(v_{2}-v_{1}\right)=v_{1}-v_{\mathrm{D}_{2}},
$$

under the assumption that $v_{\mathrm{D}_{2}}=v_{\mathrm{D}_{1}}$, can be used in (5) to obtain

$$
v_{\mathrm{m}} \stackrel{(6)}{=} \frac{1}{1+\beta}\left(v_{1}-v_{\mathrm{D}_{1}}\right)=\frac{1}{1+\beta} v_{\mathrm{ds}} .
$$

Hence, the proposed OVMC corrects the offset on $v_{1}$, i.e. $v_{\mathrm{D} 1}$, producing an output voltage $v_{\mathrm{m}}$ referred to the source potential of the DUT, exactly proportional to $v_{\mathrm{ds}}$ during its on-state.

In case the scaling of $v_{\mathrm{ds}}$ obtained by means of the voltage dividers formed by $R_{1 \mathrm{a}}-R_{2 \mathrm{a}}$ and $R_{1 \mathrm{~b}}-R_{2 \mathrm{~b}}$ is not needed, they can be bypassed (i.e. $R_{2, \mathrm{a}}=0 \Omega, R_{1, \mathrm{~b}}$ and $R_{2, \mathrm{~b}}$ removed). Consequently $\mathrm{Op}_{1}$ results unnecessary as well (Fig. 4(c)) and the system of equations given in (3) reduces to

$$
v_{\mathrm{m}}=v_{1}-\frac{R_{4}}{R_{3}}\left(v_{2}-v_{1}\right) \stackrel{(6)}{=} v_{\mathrm{ds}} .
$$

The degree of freedom given by $\beta$ is lost, i.e. the input voltage range of the circuit is reduced, but the number of required components is halved.

To provide a better understanding of the proposed OVMC, additional considerations are herein reported to conclude the section. As in the diode-based circuit presented in Fig. 2(b), the values of $V_{\mathrm{p}}$ and $R_{6}$ fix the current in $\mathrm{D}_{1}$ and $\mathrm{D}_{2}$ during on-state. $V_{\mathrm{p}}=10 \mathrm{~V}$ (Section III-B) and $R_{6}=750 \Omega$ (Section II) are selected. If the currents flowing in the voltage dividers formed by $R_{1 \mathrm{a}}-R_{2 \mathrm{a}}$ and $R_{1 \mathrm{~b}}-R_{2 \mathrm{~b}}$ are negligible (see Fig. 4(a)), the operating point of the OVMC when $\mathrm{T}_{2}$ is in on-state is defined by

$$
i_{\mathrm{p}}=\frac{V_{\mathrm{p}}-v_{\mathrm{D}_{2}}-v_{\mathrm{D}_{1}}-v_{\mathrm{ds}}}{R_{6}} .
$$

If $v_{\mathrm{ds}}=0 \mathrm{~V}$ and $v_{\mathrm{D}_{1}}=v_{\mathrm{D}_{2}}=1 \mathrm{~V}$ are assumed, $i_{\mathrm{p}} \approx 10 \mathrm{~mA}$ $\doteq I_{\mathrm{p} \text {,nom }}$. Consequently, $P_{\mathrm{p} \text {,nom }}=V_{\mathrm{p}} I_{\mathrm{p} \text {,nom }} \approx 100 \mathrm{~mW}$ defines the power consumption of the OVMC from the main power source. It is important to limit $i_{\mathrm{p}}$ to a negligible fraction, e.g. $1 \%$, of the current flowing through $\mathrm{T}_{2}$ to avoid an increase and/or distortion of the OV (and to limit $P_{\mathrm{p}, \text { nom }}$ ). This limit is strictly application dependent and, if exceeded, the additional OV should at least be considered in the calculation of $R_{\mathrm{ds}, \mathrm{on}}$. Moreover, a lower boundary of $i_{\mathrm{p}}$ is also set from the parasitic currents circulating in the OVMC, e.g. in the voltage divider formed by $R_{1 \mathrm{a}}-R_{2 \mathrm{a}}$ and in the input of $\mathrm{Op}_{2}$. If $i_{\mathrm{p}}$ is reduced below a certain threshold, it would result impossible to neglect them and the fundamental assumption $i_{\mathrm{D}_{1}}=i_{\mathrm{D}_{2}}$ would be violated, compromising the accuracy of the measurement. $V_{\mathrm{p}}$ defines as well, together with $Z_{1}$, the value of $v_{\mathrm{ds}}$ at which the circuit snaps. The blocking voltage of $Z_{1}$ is selected to be bigger than $V_{\mathrm{p}}$ to avoid its conduction during on-state of $\mathrm{T}_{2}$, but on the other hand low enough to limit $v_{1}$ during off-state. Consequently the sole $D_{1}$ blocks the offstate voltage $V_{\mathrm{DC}}$, protecting the measurement circuitry.

Finally, in order to facilitate the modular integration of the 


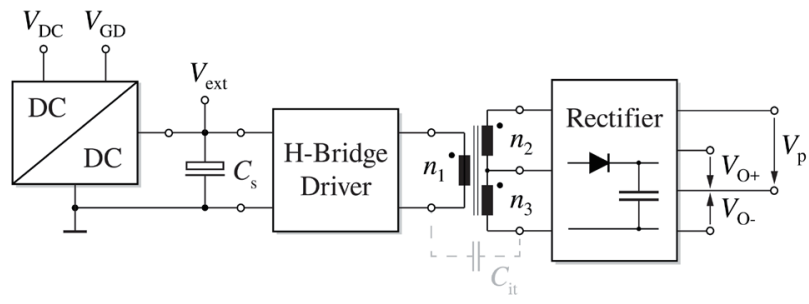

Fig. 5. Schematic representation of the OVMC supply circuitry. $V_{\mathrm{p}}, V_{\mathrm{O}+}$ and $V_{\mathrm{O}^{-}}$can be generated from one of the supply voltages available in the power converter, e.g. $V_{\mathrm{DC}}$ and $V_{\mathrm{GD}}$, facilitating the integration of the OVMC. Galvanic isolation is guaranteed and a small value of parasitic capacitance $C_{\mathrm{it}}$ ensures high common-mode rejection ratio.

OVMC in a power converter (e.g. towards intelligent gate drivers), Fig. 5 illustrates different options for the generation of the required supply voltages. In the proposed solution, $V_{\mathrm{p}}$ and the supply voltages of the operational amplifiers $V_{\mathrm{O}+}$ and $V_{\mathrm{O}-}=-V_{\mathrm{O}+}$ are galvanic isolated and generated from the gate driver supply voltage $V_{\mathrm{GD}}$. A small value of parasitic capacitance $C_{\text {it }}$ ensures high common-mode rejection ratio. Thanks to the isolated supplies, the proposed OVMC can be connected, without any additional precaution, as well to the high-side power semiconductor $\mathrm{T}_{1}$ (Fig. 1). Hence, the $\mathrm{OV}$ of $\mathrm{T}_{1}$ can be measured with respect to its source potential, i.e. $v_{\mathrm{ds}}$. To avoid a differential measurement and common mode disturbances, it can be convenient to refer the OVM to a constant voltage, e.g. $V_{\mathrm{DC}}$. To do so, the schematic of the proposed OVMC should be mirrored and referred to the drain potential of $\mathrm{T}_{1}$. Afterwards, all the calculations performed for the low-side case remain valid.

\section{B. Accuracy Measurement and DC Calibration}

The accuracy of the proposed OVMC with respect to the assumptions made in Section III-A is herein verified and calibration measurements support the analysis.

The first requirement for a correct operation of this OVMC is based on the identity $v_{\mathrm{D}_{2}}=v_{\mathrm{D}_{1}}$. First, in order to guarantee $i_{\mathrm{D}_{1}}=i_{\mathrm{D}_{2}}$, the current flowing in the voltage divider formed by $R_{1, \mathrm{a}}-R_{1, \mathrm{~b}}$ (if present) must be negligible. If this cannot be achieved by increasing the value of $R_{1, \mathrm{a}}+R_{1, \mathrm{~b}}$, an operational amplifier $\mathrm{Op}_{3}$ can be added between $v_{1}$ and $R_{1 \mathrm{a}}$.

However, even if $i_{\mathrm{D}_{1}}=i_{\mathrm{D}_{2}}$, the eventually different junction temperatures of the diodes (i.e. $T_{\mathrm{D}_{1}}$ and $T_{\mathrm{D}_{2}}$ respectively) and their manufacturing variability can cause a mismatch of $v_{\mathrm{D}}$.

$T_{\mathrm{D}_{1}}$ can generally be higher than $T_{\mathrm{D}_{2}}$ because $\mathrm{D}_{1}$ is exposed to a wide input voltage excitation and is also physically closer to $\mathrm{T}_{2}$, where the major losses, i.e. heat, are dissipated. In order to minimize the problem, the nominal operating point of the circuit can be tuned at a temperature-independent point of the $V-I$ characteristic of the diodes (if existing and coinciding between them). Alternatively, two diodes in the same package can be chosen in order to maximize their thermal coupling. Unfortunately, as explained in Section II, several constraints already drive the selection of the diodes (e.g. small parasitic output capacitances) if good dynamic performance are required and it results difficult to find devices combining all these characteristics. More realistically, as shown in Fig. 4(b) (dashed box), a sufficient and good practice is to thermally well couple $\mathrm{D}_{2}$ with $\mathrm{D}_{1}$ on the OVMC PCB (and if necessary provide separation between the OVMC and $\mathrm{T}_{2}$ without excessively increasing the parasitic inductance of the connection). Hence, a $\Delta T_{\mathrm{D}}=T_{\mathrm{D}_{1}}-T_{\mathrm{D}_{2}}$ of only $3{ }^{\circ} \mathrm{C}$ (Fig. 2(b)) is measured in the worst-case operating conditions of interest (i.e. maximum losses of $8 \mathrm{~W}$ in $\mathrm{T}_{2}$ at the highest switching frequency of $1 \mathrm{MHz}$ and off-state voltage of $400 \mathrm{~V}$ ). In particular, depending on the operating conditions of $\mathrm{T}_{2}, T_{\mathrm{D}_{1}}$ varies between $T_{\mathrm{D}_{1, \text { min }}}=30^{\circ} \mathrm{C}$ and $T_{\mathrm{D}_{1, \max }}=40^{\circ} \mathrm{C}$.

Concerning the device variability, before assembling the circuit, two diodes featuring the same $v_{\mathrm{D}}$ in the operating conditions of interest must be selected (a variability in the range of $10 \mathrm{mV}$ is experienced in worst case among the available ones). To minimize this issue, it is convenient to reduce the variation of $i_{\mathrm{p}}$ from $i_{\mathrm{p} \text {,min }}<I_{\mathrm{p} \text {,nom }}<i_{\mathrm{p} \text {,max }}$, caused by a variation of $v_{\mathrm{ds}}$ (according to (9)). For this reason, in [12], the voltage source $V_{\mathrm{p}}-R_{6}$ is replaced with a current source $I_{\mathrm{p}}$. However, considering the experiment presented in the introduction (i.e. a specimen power MOSFET with $R_{\mathrm{ds}, \mathrm{on}}=25$ $\mathrm{m} \Omega$ conducting $\pm 20 \mathrm{~A}$ ), the excursion of $v_{\mathrm{ds}}$ is in the range of $\pm 500 \mathrm{mV}$ and the current variation results

$$
\Delta i_{\mathrm{p}}=\frac{i_{\mathrm{p}, \max }}{i_{\mathrm{p}, \min }} \stackrel{(9)}{=} \frac{V_{\mathrm{p}}-v_{\mathrm{D}_{2}}-v_{\mathrm{D}_{1}}-v_{\mathrm{ds}, \min }}{V_{\mathrm{p}}-v_{\mathrm{D}_{2}}-v_{\mathrm{D}_{1}}-v_{\mathrm{ds}, \max }} \approx 1.1 .
$$

Even a worst-case $\pm 15 \%$ variation, i.e. $\pm 1.5 \mathrm{~mA}$, is assumed not to have any influence on the OVMC accuracy. To quantitative support this statement, $v_{\mathrm{D}_{1}}$ and $v_{\mathrm{D}_{2}}$ are reported in TABLE I as a function of $i_{\mathrm{p}}$ and $T_{\mathrm{D}_{1}}$. A worst-case mismatch in the diode voltage drops $v_{\Delta \mathrm{D}}=v_{\mathrm{D}_{1}}-v_{\mathrm{D}_{2}}=2 \mathrm{mV}$ and a temperature coefficient of $1 \frac{\mathrm{mV}}{{ }^{\circ} \mathrm{C}}$ are observed. $v_{\Delta \mathrm{D}}$ must be negligible compared to the measured vds not to affect the OVM accuracy. This becomes more and more difficult to achieve at low OV levels, e.g. with $v_{\mathrm{ds}}<40 \mathrm{mV}$, and if desired an even more sophisticated diode matching and/or calibration procedure can be adopted. However, other problems arise when $v_{\mathrm{ds}}$, i.e. the DUT current, approaches $0 \mathrm{~V}$ and are discussed in Section IV-A. Finally, it can be noticed that, due to the small value of $i_{\mathrm{p}}$, the diode model provided in [27] is not valid. The proposed calibration procedure is required to enable the performance of the OVMC described in this section. Other interesting considerations regarding the precise tuning of the proposed OVMC are reported in Appendix A as guideline for its design.

To conclude, once the crucial aspects related to the design of the proposed OVM hardware are clarified and taken into account, the achieved accuracy is measured. Fig. 6 illustrates the relative error $\varepsilon_{\mathrm{r}}$ (right y-axis) from a DC input $v_{\mathrm{ds}}(x$ axis) to a DC output $v_{\mathrm{m}}$ (left $y$-axis) in a range from $-2 \mathrm{~V}$ to $2 \mathrm{~V} . \varepsilon_{\mathrm{r}}$ is confined between $\pm 2 \%$ with an absolute maximum error $\varepsilon_{\max }=5 \mathrm{mV}$. Here, and in the rest of the analysis, the gain of the proposed OVMC is normalized to 1 for the sake of clarity. 
TABLE I

Mismatch in the Diode Voltage Drops as Function of Their Temperature AND CURRENT

\begin{tabular}{ccccc}
\hline \hline $\boldsymbol{i}_{\mathbf{p}}$ & $\boldsymbol{T}_{\mathbf{D}_{\mathbf{1}}}$ & $\boldsymbol{v}_{\mathbf{D}_{\mathbf{1}}}(\mathrm{mV})$ & $\boldsymbol{v}_{\mathbf{D}_{2}}(\mathrm{mV})$ & $\boldsymbol{v}_{\Delta \mathbf{D}}(\mathrm{mV})$ \\
\hline \multirow{2}{*}{$i_{\mathrm{p}, \min }$} & $T_{\mathrm{D}_{1}, \min }$ & 831 & 832 & -1 \\
& $T_{\mathrm{D}_{1}, \max }$ & 820 & 818 & 2 \\
\hline \multirow{2}{*}{$i_{\mathrm{p}, \max }$} & $T_{\mathrm{D}_{1}, \min }$ & 838 & 839 & -1 \\
& $T_{\mathrm{D}_{1}, \max }$ & 828 & 826 & 2 \\
\hline \hline
\end{tabular}

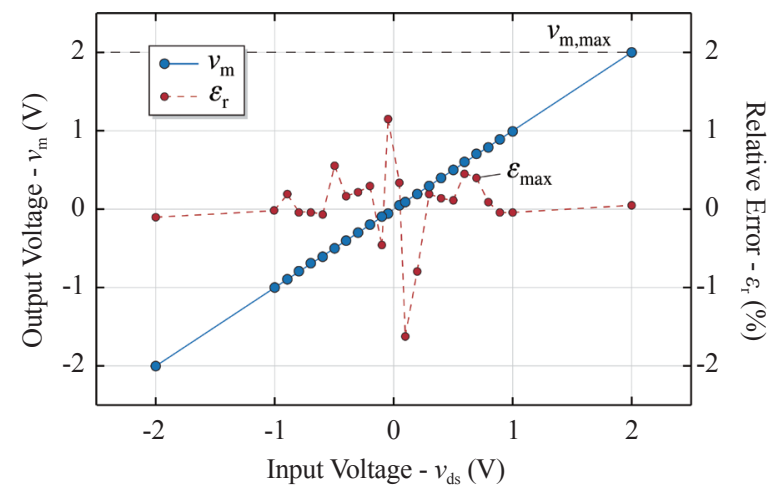

Fig. 6. Results of the DC calibration of the proposed OVMC. When a DC voltage $-2 \mathrm{~V}<v_{\mathrm{ds}}<2 \mathrm{~V}$ is applied at the input ( $x$-axis), the output $v_{\mathrm{m}}$ (left $y$-axis) deviates less than $5 \mathrm{mV}$, corresponding to $\varepsilon_{\mathrm{r}}<2 \%$ (right $y$-axis) in worst-case.

\section{Bandwidth Measurement and AC Calibration}

A severe bandwidth requirement is mandatory in all the application areas of OVMCs mentioned in the introduction when fast switching semiconductors are considered. The bandwidth and the accuracy in AC operation of the proposed OVMC are verified in this section to evaluate its applicability in the conditions of interest. Measurements are preceded from a brief discussion pointing out the most significant aspects enabling its performance.

Accurately sensing a high-frequency signal with a conventional oscilloscope probe (as in Fig. 2(a)-(b)) results in a challenge. In fact, when the high-impedance input (i.e. $1 \mathrm{M} \Omega$ ) of the oscilloscope is used, precise tuning of the probe internal capacitance is required to have a flat gain for all the frequency range of interest. Hence, the $50 \Omega$ input of the oscilloscope is preferred. $R_{5} \approx 50 \Omega$ is added at the output of the proposed OVMC (cf. Fig. 4(a)-(c)) to match the output impedance of $\mathrm{Op}_{2}$ with the characteristic impedance of the used cable (i.e. $50 \Omega$ ). This is possible only given the presence of $\mathrm{Op}_{2}$, driving the necessary output current in the oscilloscope without affecting the circuit performance. Therefore, the selection range of operational amplifiers is limited from their output current and voltage capabilities. Among the available devices, a low-noise $1 \mathrm{GHz} 10 \mathrm{~V} 40 \mathrm{~mA}$ operational amplifier [28] is selected to maximize the achievable bandwidth of the OVMC.

The results of the high-frequency calibration are described in Fig. 7. Three different triangular waveforms at $700 \mathrm{kHz}$

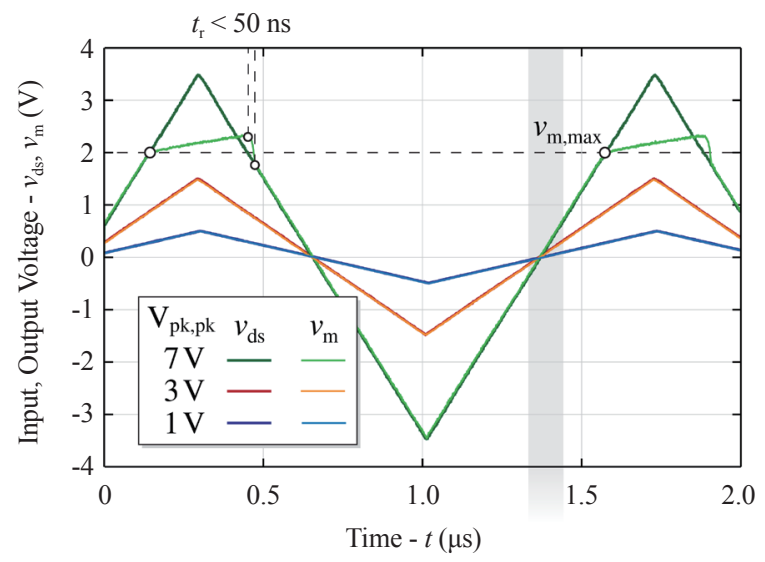

(a)
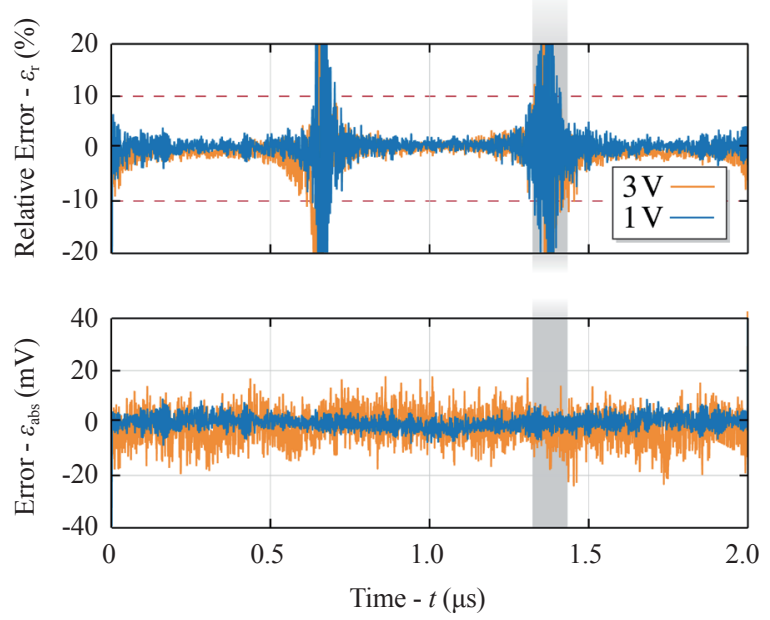

(b)

Fig. 7. Results of the AC calibration of the proposed OVMC. When AC voltage waveforms of different amplitudes are applied at the input $v_{\mathrm{ds}}$, the output waveforms $v_{\mathrm{m}}$ are practically indistinguishable from them: $\varepsilon_{\mathrm{abs}}$ is limited by the oscilloscope resolution.

with peak-to-peak voltage amplitudes $\mathrm{V}_{\text {pk,pk }}$ increasing from $1 \mathrm{~V}$ to $7 \mathrm{~V}$ are generated at the input $v_{\mathrm{ds}}$ and plotted (blue, red and dark green) on top of the measured output $v_{\mathrm{m}}$ (sky blue, orange and light green) in Fig. 7(a). When $v_{\mathrm{ds}}<2 \mathrm{~V}$ the input and output waveforms are indistinguishable. At $v_{\mathrm{ds}}=$ $v_{\mathrm{m}, \max }=2 \mathrm{~V}, \mathrm{Op} \mathrm{p}_{1}$ saturates and $v_{\mathrm{m}}$ (light green) is distorted. In Fig. 7(b), the relative $\varepsilon_{\mathrm{r}}$ and absolute $\varepsilon_{\mathrm{abs}}$ errors are reported. $\varepsilon_{\text {abs }}$ is limited between $\varepsilon_{\text {abs;max }}= \pm 10 \mathrm{mV}$ in the worst-case of $3 \mathrm{~V}_{\text {pk:pk }}$ and has a zero mean. It must be mentioned that with a 10 bits oscilloscope on a $8 \mathrm{~V}$ vertical window, $\varepsilon_{\text {abs;max }}$ is in the range of the oscilloscope resolution $\left(8 \mathrm{~V} / 2^{10} \approx 8 \mathrm{mV}\right) . \varepsilon_{\mathrm{r}}$ is, for the same reason, mainly in a $\pm 5 \%$ range; however, when $v_{\mathrm{ds}}$ approaches zero (gray shaded area), the division between two small numbers causes a numerical issue and $\varepsilon_{\mathrm{r}}$ diverges.

Finally, with a vector network analyzer, the bandwidth of the proposed OVMC is measured exciting it at the input $v_{\mathrm{ds}}$ with sinusoidal waveforms up to $2 \mathrm{~V}_{\mathrm{pk}, \mathrm{pk}}$. The $-3 \mathrm{~dB}$ bandwidth is outside the measurable frequency range of the instrument (i.e. $50 \mathrm{MHz}$ ) while the more intuitive normalized linear gain $g_{\text {lin }}$ is 1.0 until $1 \mathrm{MHz}$ and still 0.97 at $10 \mathrm{MHz}$, 
TABLE II

Specifications of the Power Semiconductors Considered as DUT for the Proposed OVMC

\begin{tabular}{cccccll}
\hline \hline Specimen & Type & $V_{\mathrm{dc}, \max }(\mathrm{V})$ & $I_{\mathrm{ds}, \max }(\mathrm{A})$ & $R_{\mathrm{d}, \text { on }}(\mathrm{m} \Omega)$ & Package & \multicolumn{1}{c}{ Figures } \\
\hline \multirow{2}{*}{$\mathrm{A}$} & SiC Power MOSFET & 1200 & 98 & 25 & TO-247-3 & $14(\mathrm{a}), 13,11$ and 9 \\
$\mathrm{~B}$ & Si Super-Junction MOSFET & 700 & 46 & 40 & TO-247 & $14(\mathrm{~b})$ and 10 (b) \\
$\mathrm{C}$ & E-mode GaN HEMT & 650 & 30 & 50 & GaNPX & $14(\mathrm{c}), 10(\mathrm{a})$ and 3 \\
\hline \hline
\end{tabular}

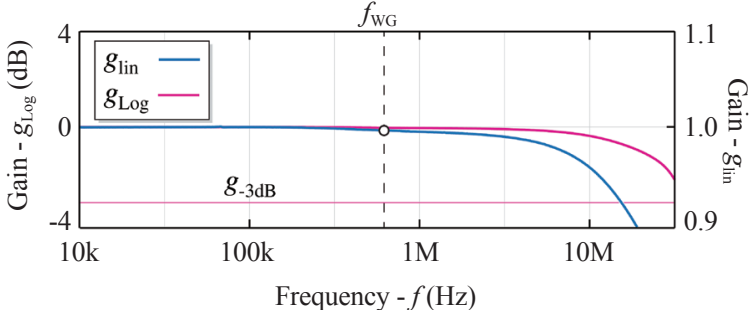

Fig. 8. Measured bandwidth of the proposed OVMC. The $-3 \mathrm{~dB}$ bandwidth is above $50 \mathrm{MHz}$ (magenta) while $g_{\text {lin }}=1.0$ until $1 \mathrm{MHz}$ and 0.97 at 10 $\mathrm{MHz}$ (blue).

independently of the input signal amplitude (Fig. 8). In order to guarantee the linearity in these measurements, the input signal never exceeds $v_{\text {mmax }}$. The real bandwidth of the circuit is, instead, strongly influenced from its dynamic response after the DUT turn-on switching transition, which is the topic of the next section.

\section{Dynamic Response}

To conclude the characterization of the proposed OVMC, its outstanding dynamic response is analyzed in this section, accompanied by a discussion on the main features enabling this achievement.

As mentioned, it is important to perform accurate OVMs immediately after the turn-on transition of the DUT. This allows to capture eventual dynamic $R_{\mathrm{ds} \text { on }}$ effects, the diode conduction phase during dead-times and enables high-frequency measurements necessary in all the mentioned application areas. In fact, the time constant of the dynamic response must be negligible compared to the duration of the DUT on-state to obtain meaningful OVMs.

In Fig. 9, the dynamic response of the proposed OVMC is compared with the one of a state-of-the-market (SoM) commercial OVM probe. The two measured waveforms highlight the faster response of the proposed approach (blue). The measured signal is the on-state resistance $r_{\mathrm{ds}, o n}(\mathrm{t})$ (time dependency is omitted from now on) of a commercial $1200 \mathrm{~V}$ $\mathrm{SiC}$ Power MOSFET (Specimen A in TABLE II) for two different case temperatures. The value of $r_{\mathrm{ds}, \text { on }}$ is obtained dividing the output of the OVMCs $v_{\mathrm{m}}$ by the DUT current $i_{\mathrm{sw}}$ (see Fig. 1) and must be limited to the time window where the DUT is in on-state (i.e. after $t_{0}$ ). The key design guidelines at the basis of the achieved performance rely on the selection of high bandwidth operational amplifiers and diodes with small parasitic capacitance, and on a low inductive PCB design (especially concerning the commutation loop of $\mathrm{D}_{1}$ ). In

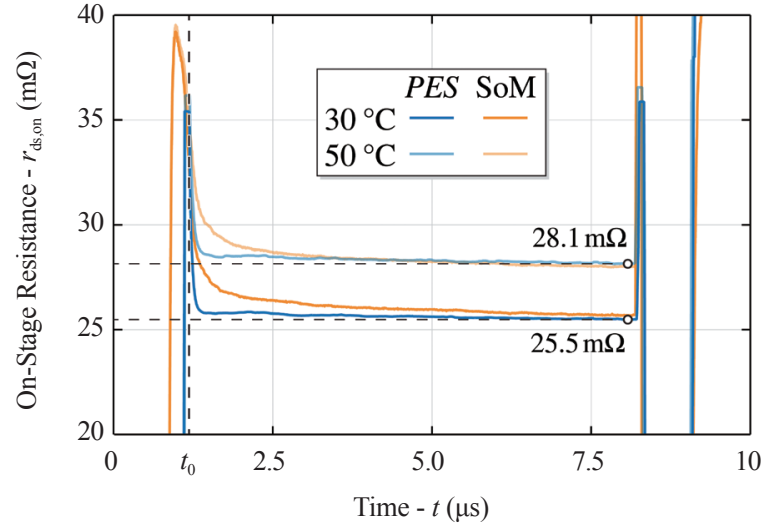

Fig. 9. Comparison in terms of dynamic response between the proposed OVMC (PES) and a state-of-the-market (SoM) commercial OVM probe. When the DUT turns on at $t_{0}$, the output of the proposed OVMC immediately measures the correct $\mathrm{OV}$, i.e. the nominal $r_{\mathrm{ds}, o n}$ value, whereas the commercial device features a time constant in the $\mu$ s-range. E.g. a fast dynamic response increases the maximum switching frequency at which an OVMC can perform useful measurements, since the dynamic transient must be negligible compared to the duration of the DUT on-state.

order to quantify the dynamic response time of the proposed OVMC, two more measurements, showing the turn-on transition of different DUTs, are performed (Fig. 10). In Fig. 10(a), a commercial 650V E-mode GaN HEMT (Specimen $\mathrm{C}$ in TABLE II) is tested. The three voltage measurements are $v_{\mathrm{ds}}$ (yellow), $v_{\mathrm{gs}}$ (green) and $v_{\mathrm{m}}$ (blue). When the highside transistor $\mathrm{T}_{1}$ is turned off, $v_{\mathrm{ds}}$ drops from $V_{\mathrm{DC}}$ to $0 \mathrm{~V}$ because of a positive load current $i_{\text {load }}$ (not shown, see Fig. 1). The anti-parallel body diode of $\mathrm{T}_{2}$ (i.e. $\mathrm{D}_{\mathrm{T}_{2}}$ ) immediately conducts and $v_{\mathrm{m}}=-V_{\mathrm{fv}, \mathrm{BD}}=-7 \mathrm{~V}$ is clamped to the bottom of the oscilloscope screen. When $v_{\mathrm{gs}}$ reaches $V_{\mathrm{th}, \mathrm{T}_{2}}$ (green dot), $\mathrm{T}_{2}$ turns on and $i_{\mathrm{sw}}$ commutates from $\mathrm{D}_{\mathrm{T}_{2}}$ to the channel of $\mathrm{T}_{2}$. Consequently $v_{\mathrm{m}}$ changes from the forward voltage of $\mathrm{D}_{\mathrm{T} 2}$ (i.e. $\left.-V_{\mathrm{fv}, \mathrm{BD}}\right)$ to $r_{\mathrm{ds}, 0 n} i_{\mathrm{sw}}$. As the white cursors highlight, the response time of the proposed OVMC is less than $50 \mathrm{~ns}$ if the real transition is assumed instantaneous.

The same situation is reproduced in Fig. 10(b) with a commercial Si Super-Junction MOSFET (Specimen B in TABLE II). In this condition, $V_{\mathrm{fv}, \mathrm{BD}} \approx 0.8 \mathrm{~V}$ can be accurately measured during all the conduction time of $\mathrm{D}_{\mathrm{T}_{2}}$. In transparency a second measurement with a shorter dead-time is overlapped.

\section{Online Conduction Loss Measurement}

In this section, the integration of the proposed OVMC in a power converter (Fig. 1) is commented and the OV, i.e. $R_{\mathrm{ds}, o n}$, 


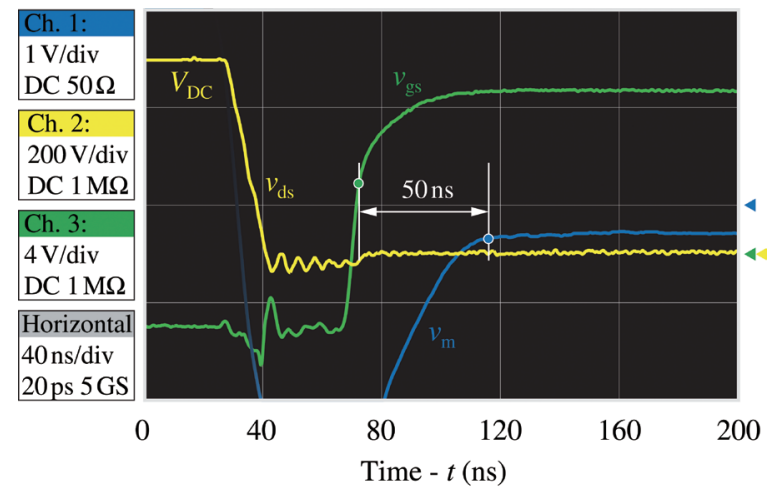

(a)

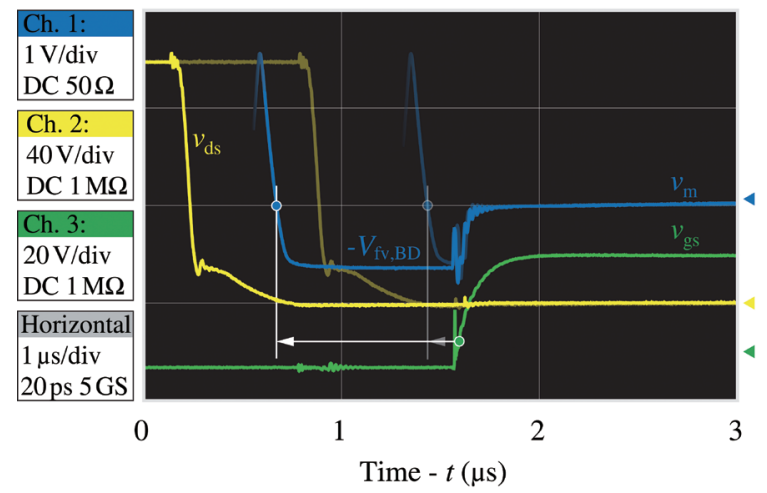

(b)

Fig. 10. Analysis of the dynamic response of the proposed OVMC. (a) less than $50 \mathrm{~ns}$ after $v_{\mathrm{gs}}$ has reached $V_{\text {th }}$ (green dot), $v_{\mathrm{m}}$ (blue) is settled to the correct value, even in the case of significant $V_{\mathrm{fv}, \mathrm{BD}}$, e.g. $>2 \mathrm{~V}$. (b) when $V_{\mathrm{fv}, \mathrm{BD}}$ is in the measurable range, the proposed OVMC allows to monitor the conduction time of $\mathrm{D}_{\mathrm{T}_{2}}$, evaluating the dead-time conduction losses and its effective duration.

of different power semiconductors is measured as function of their operating conditions. The measurement setup is initially described. The associated challenges are then addressed and the proposed solutions are ultimately commented. Finally, since the original reason motivating the development of this OVMC is the improvement of the measurement accuracy of calorimetric switching loss measurement methods, the presented results are discussed accordingly.

\section{A. Conduction Loss Measurement Setup}

The setup is equivalent to the schematic shown in Fig. 1. Assuming symmetrical triangular current mode (TCM) operation of the half-bridge (i.e. $\mathrm{T}_{1}$ and $\mathrm{T}_{2}$ are operated with complementary $50 \%$ duty cycles and no load is connected), the analysis can be limited to the conduction time of $\mathrm{T}_{2}$. All the relevant measured waveforms are shown in Fig. 11 (a)Fig. 11(b). In particular, the switch node voltage $v_{\mathrm{ds}}$ (blue in Fig. 11 (a)), the load current $i_{\text {load }}$ (orange), the gate voltage $v_{\mathrm{gs}}$ (green, $V_{\mathrm{gs}, \mathrm{ON}}=18 \mathrm{~V}$ and $V_{\mathrm{gs}, \mathrm{OFF}}=-5 \mathrm{~V}$ ) and the output of the proposed OVMC $v_{\mathrm{m}}$ (blue in Fig. 11(b)). Inside the time window $t_{1}-t_{2}$, the on-state resistance $r_{\mathrm{ds}, \text { on }}$ of $\mathrm{T}_{2}$ can be determined dividing $v_{\mathrm{m}}$ by $-i_{\text {load }}=i_{\text {sw }}$ (Fig. 11(c)). The instantaneous conduction losses pcond can be calculated as $r_{\mathrm{ds}, \mathrm{on}} i_{\mathrm{sw}}^{2}$

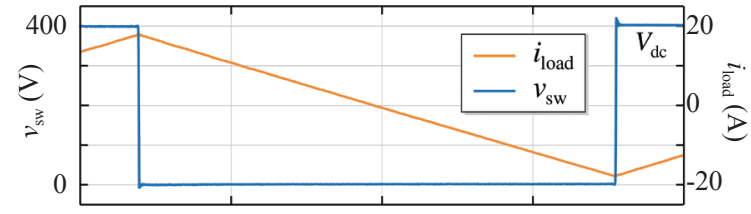

(a)

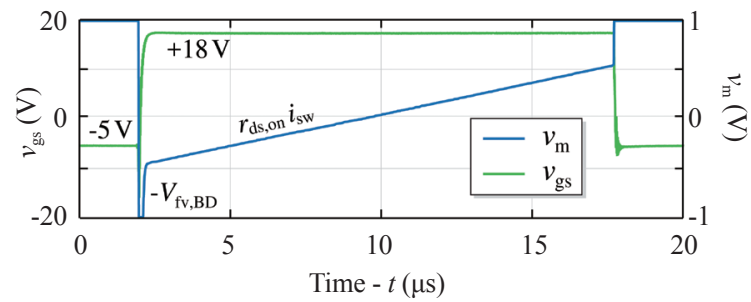

(b)

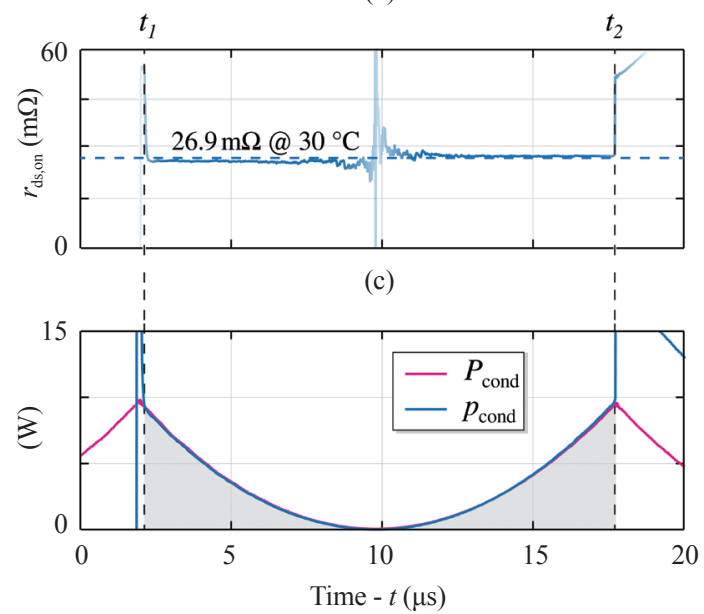

(d)

Fig. 11. Typical waveforms (a)-(b) measured on the half-bridge (Fig. 1) operating in triangular current mode (TCM). When $v_{\mathrm{gs}}=V_{\mathrm{gs}, \mathrm{ON}}$ (green), $\mathrm{T}_{2}$ conducts and $v_{\mathrm{m}}$ (blue) is proportional to $i_{\mathrm{sw}}$ (orange). In (c) $r_{\mathrm{ds}, \text { on }}$ is calculated between $\mathrm{t}_{1}$ and $\mathrm{t}_{2}$ dividing $v_{\mathrm{m}}$ by $i_{\mathrm{sw}}$, while in (d) $p_{\text {cond }}$ is obtained multiplying $v_{\mathrm{m}}$ and $i_{\mathrm{sw}}$

or equivalently $v_{\mathrm{m}} i_{\mathrm{sw}}$ (blue in Fig. 11(d)). Finally, the average conduction losses can be obtained as average of $p_{\text {cond }}$ during on-state of $\mathrm{T}_{2}$ (gray shaded area).

Similarly, an average $R_{\mathrm{ds}, \mathrm{on}}$ value can be obtained as average of $r_{\mathrm{ds}, \text { on }}$. In this case, $R_{\mathrm{ds}, \mathrm{on}}=26.9 \mathrm{~m} \Omega$ (Fig. 11(c)) results for Specimen A in TABLE II. Consequently, the approximated conduction losses $P_{\text {cond }}$ can be directly calculated as $R_{\mathrm{ds}, \mathrm{on}} i_{\mathrm{sw}}^{2}$ (magenta in Fig. 11(d)). Given the almost perfect overlap between the blue and magenta curves in Fig. 11(d), when $p_{\text {cond }}$ and $P_{\text {cond }}$ are averaged, $3.6 \mathrm{~W}$ results in both cases. However, in general, the first approach is preferred, since it takes into account dynamic $R_{\mathrm{ds}, o n}$ effects and the eventual current dependency of $R_{\mathrm{ds}, \mathrm{on}}$.

In order to perform accurate measurements in this setup and operating conditions, two challenges, associated in particular with the reduced OV and high current slopes that needs to be measured, are faced. The first challenge to overcome is represented by the parasitic inductance $L_{\mathrm{P}}$ (Fig. 1) in series with $\mathrm{T}_{2}$. Fig. 12(a) highlights the load current slope $\mathrm{d} i_{\text {load }} / \mathrm{d} t$ $=-\mathrm{d} i_{\text {sw }} / \mathrm{d} t$ (up to tens of $\mathrm{A} / \mu \mathrm{s}$ ) that in combination with $L_{\mathrm{P}}$ causes a voltage offset (red in Fig. 12(b)) on $v_{\mathrm{m}}$ according to 


$$
v_{\mathrm{m}}=R_{\mathrm{ds}, \mathrm{on}} i_{\mathrm{sw}}+L_{\mathrm{P}} \frac{\mathrm{d} i_{\mathrm{sw}}}{\mathrm{d} t}
$$

(blue in Fig. 12(b)). Hence, calculating $r_{\mathrm{ds}, \mathrm{on}}$ as

$r_{\mathrm{ds}, \text { on }}=\frac{v_{\mathrm{m}}}{i_{\mathrm{sw}}} \stackrel{(11)}{=} R_{\mathrm{ds}, \text { on }}+\frac{L_{\mathrm{P}}}{t}=R_{\mathrm{ds}, \text { on }}\left(1+\frac{\tau_{\mathrm{RL}}}{t}\right)$

the term $\frac{\tau_{\mathrm{RL}}}{t}\left(\tau_{\mathrm{RL}} \doteq \frac{L_{\mathrm{P}}}{R_{\mathrm{ds}, \text { on }}}\right)$ distorts the result. Therefore, in contrast to a constant $R_{\mathrm{ds}, \mathrm{on}}$ (dashed in Fig. 12(c)), the waveform of $r_{\mathrm{ds}, \text { on }}$, solid in Fig. 12(c), is obtained. E.g. if $L_{\mathrm{P}}$ $=8 \mathrm{nH}$ and $R_{\mathrm{d} \text {,on }}=50 \mathrm{~m} \Omega$ then $\tau_{\mathrm{RL}} \approx 150 \mathrm{~ns}$. Hence, after $t=$ $5 \tau_{\mathrm{RL}}=800 \mathrm{~ns}, r_{\mathrm{ds}, \text { on }}=1.2 R_{\mathrm{ds}, \text { on }}=60 \mathrm{~m} \Omega$. From (12) it can be concluded that $L_{\mathrm{P}}$ should be minimized, i.e. the connection from the DUT to the OVMC should start as close as possible from the drain and source terminals of $\mathrm{T}_{2}$, excluding any additional path where $i_{\mathrm{sw}}$ flows. Unfortunately, part of $L_{\mathrm{P}}$ is located internally in the package of the DUT and no design expedient results helpful. A compensation network could be inserted and tuned, however it would negatively affect the dynamic performance of the proposed OVMC. Alternatively, the inductive voltage drop $v_{\mathrm{m}, \mathrm{o}}$ can be isolated from the resistive one taking advantage of the zero crossing of $i_{\text {sw. }}$.

I.e., measuring $v_{\mathrm{m}}$ and $\mathrm{d} i_{\mathrm{sw}} / \mathrm{d} t$ when $i_{\mathrm{sw}}=0 \mathrm{~A}$, (11) can be solved for $L_{\mathrm{P}}$. Formally

$$
\left.v_{\mathrm{m}, \mathrm{o}} \doteq v_{\mathrm{m}}\right|_{i_{\mathrm{sw}}=0} \stackrel{(11)}{=} L_{\mathrm{P}} \frac{\mathrm{d} i_{\mathrm{sw}}}{\mathrm{d} t} .
$$

Repeating this measurement for different $\mathrm{d} i_{\mathrm{sw}} / \mathrm{d} t, L_{\mathrm{P}}$ can be calculated as average of several

$$
L_{\mathrm{P}_{\mathrm{i}}}=\frac{v_{\mathrm{m}, \mathrm{o}_{\mathrm{i}}}}{\mathrm{d} i_{\mathrm{sw}_{\mathrm{i}}} / \mathrm{d} t}
$$

and its value can be used to adjust $v_{\mathrm{m}_{\mathrm{i}}}$. From the practical point of view, this results in subtracting $v_{\mathrm{m}, \mathrm{o}_{\mathrm{i}}}$ from $v_{\mathrm{m}_{\mathrm{i}}}$ in each measurement, ensuring $v_{\mathrm{m}_{\mathrm{i}}}=0 \mathrm{~V}$ when $i_{\mathrm{sw}_{\mathrm{i}}}=0 \mathrm{~A}$. Whereas this assumption sounds legit and sufficient by itself, calculating $L_{\mathrm{P}}$ as in (14) provides a physical motivation to this adjustment. Moreover, obtaining constant $L_{\mathrm{P}_{\mathrm{i}}}$ across different measurements guarantees their correctness. This becomes more and more important when the zero crossing of $i_{\mathrm{sw}}$ is not present and/or measurable and the knowledge of $L_{\mathrm{P}}$ is the only option to correct the measurement result. Alternatively, $r_{\mathrm{ds}, \text { on }}$ can be calculated as the ratio between $\mathrm{d} v_{\mathrm{m}} / \mathrm{d} t$ and $\mathrm{d} i_{\mathrm{sw}} / \mathrm{d} t$. Even if not affected from $v_{\mathrm{m}, \mathrm{o}}$, this approach loses accuracy when the voltage and current slopes become flatter.

The second challenge is easily highlighted applying the propagation of uncertainty rule on $r_{\mathrm{ds}, \mathrm{on}}=v_{\mathrm{m}} / i_{\mathrm{sw}}$ :

$$
\begin{aligned}
\sigma_{r_{\mathrm{ds}, \text { on }}} & =\sqrt{\left(\frac{\partial r_{\mathrm{ds}, \mathrm{on}}}{\partial v_{\mathrm{m}}} \sigma_{v_{\mathrm{m}}}\right)^{2}+\left(\frac{\partial r_{\mathrm{ds}, \mathrm{on}}}{\partial i_{\mathrm{sw}}} \sigma_{i_{\mathrm{sw}}}\right)^{2}} \\
& =\frac{1}{i_{\mathrm{sw}}} \sqrt{\sigma_{v_{\mathrm{m}}}^{2}+\left(r_{\mathrm{ds}, \mathrm{on}} \sigma_{i_{\mathrm{sw}}}\right)^{2}}
\end{aligned}
$$

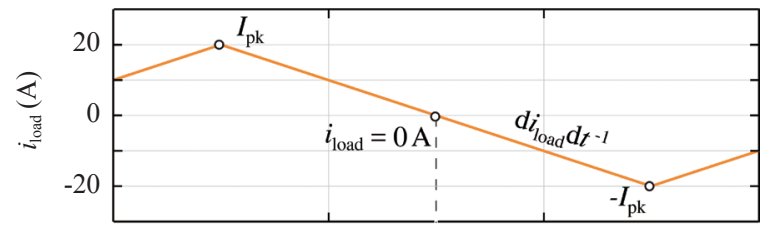

(a)

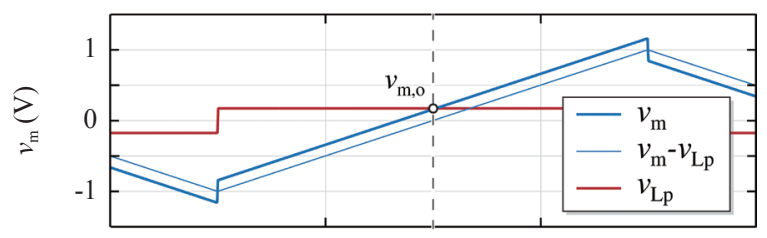

(b)

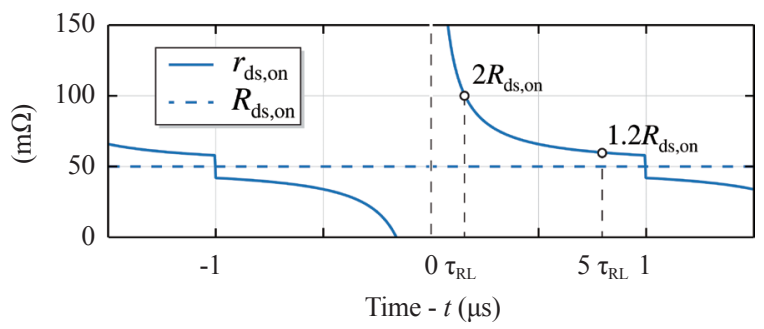

(c)

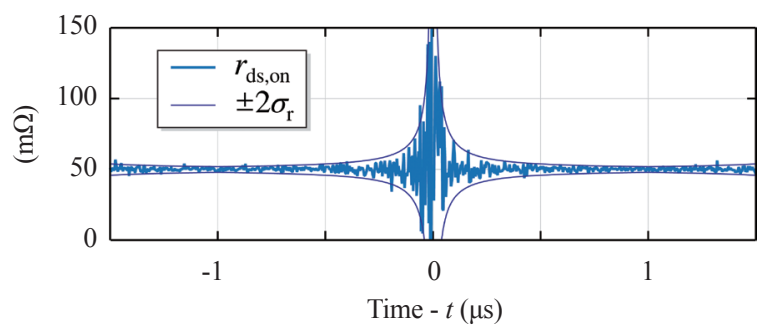

(d)

Fig. 12. Comparison of real and ideal waveforms simulated on the halfbridge (Fig. 1) operating in TCM. The combination of $L_{\mathrm{P}}$ and $\mathrm{d} i_{\mathrm{sw}} / \mathrm{d} t$ causes a distortion on $v_{\mathrm{m}}$ (b) and consequently on $r_{\mathrm{ds}, \mathrm{on}}$ (c). Moreover, a measurement error on $v_{\mathrm{m}}$ translates into an error on $r_{\mathrm{ds}, \text { on }}(\mathrm{d})$ according to (16), particularly amplified for small $i_{\mathrm{sw}}$ as in (15).

and

$$
\sigma_{\% r_{\mathrm{ds}, \text { on }}}=\frac{\sigma_{r_{\mathrm{ds}, \text { on }}}}{r_{\mathrm{ds}, \text { on }}} \stackrel{\stackrel{15}{=}}{=} \frac{\sigma_{v_{\mathrm{m}}}}{r_{\mathrm{ds}, \text { on }} i_{\mathrm{sw}}}=\sigma_{\% v_{\mathrm{m}}} .
$$

$\sigma x$ and $\sigma \% x$ indicate the absolute and relative error on the measured quantity $x$, respectively. (15) proves why $\sigma_{r_{\mathrm{d} s, 0 n}}$ and therefore $r_{\mathrm{ds}, \mathrm{on}}$ diverges when $i_{\mathrm{sw}}$ approaches zero (see Fig. 11(c) and Fig. 12(c)-(d)). (16), instead, expresses how $\sigma \%_{r_{\mathrm{d}, \text { on }}}$ coincides with $\sigma \%_{v_{\mathrm{m}}}$ when $\sigma_{\mathrm{i}_{\mathrm{sw}}}=0$. In other words, any error on $v_{\mathrm{m}}$ reflects one-to-one (relatively) on $r_{\mathrm{ds}, \text { on }}$ (i.e. on $p_{\text {cond }}$ ). An example of $r_{\mathrm{d} s, \mathrm{on}}$, corrected from $v_{\mathrm{m}, \mathrm{o}}$, but resulting from $v_{\mathrm{m}}$ with $\sigma_{v_{\mathrm{m}}}=5 \mathrm{mV}$ to highlight the effect, is shown in Fig. 12(d). Since the only expedient to minimize both phenomena described from (15) and (16) is to reduce $\sigma_{v_{\mathrm{m}}}$, this section clearly justifies the effort placed on the accuracy analysis of the proposed OVMC addressed in Section III. Moreover, it highlights how measurements of low $r_{\mathrm{ds}, \text { on }}$ and/or high $\mathrm{d} i_{\text {load }}=\mathrm{d} t$ (e.g. wide bandgap semiconductors) introduce new 
challenges in the OVMs.

\section{B. Conduction Loss Measurement Results}

Several measurements are performed with the proposed OVMC analyzing different DUTs in different operating conditions. The results are commented in this section.

Fig. 13 compares the nominal value of $R_{\mathrm{ds}, \text { on }}$ reported in the datasheet of Specimen A in TABLE II with the values of $R_{\mathrm{ds}, \text { on }}$ measured with the proposed OVMC during double pulse test (DPT) operation (in the same conditions described in the datasheet). The black dashed line $R_{\text {data }}$ is plotted as function of the junction temperature $T_{\mathrm{j} \text {,data }}$ (i.e. bottom $x$-axis) while the blue measurement points $R_{\mathrm{DPT}}$ are plotted as function of the measured case temperature $T_{\mathrm{c}, \mathrm{DPT}}$ (i.e. top $x$-axis). Since the DPT has electric dynamics which are assumed to be faster than the thermal dynamics of the DUT, $T_{\mathrm{c}, \mathrm{DPT}} \approx$ $T_{\mathrm{j}, \mathrm{DPT}}$ is considered and the two $x$-axis coincide (i.e. $T_{\mathrm{j}, \mathrm{DPT}} \approx$ $\left.T_{\mathrm{j} \text {,data }}\right) . R_{\mathrm{DPT}}$, measured with the proposed OVMC as described in Fig. 9, match $R_{\text {data }}$ with an approximation of $\pm 3 \%$ (blue confidence bar is $\pm 5 \%$ ). The discrepancy can be attributed mainly to the device manufacturing variability. However, the results are satisfactory and confirm the performance of the proposed OVMC.

Fig. 14(a), (b) and (c), instead, summarize the values of $R_{\mathrm{ds}, \text { on }}$ measured in two different continuous operating conditions for all the Specimens of TABLE II. In particular, the orange points $R_{\mathrm{TCM}}$ are measured (with the proposed OVMC) in the conditions described in Section IV-A (i.e. TCM operation with $V_{\mathrm{DC}}=400 \mathrm{~V}, I_{\mathrm{pk}}=20 \mathrm{~A}$ and $f_{\mathrm{sw}}=30 \mathrm{kHz}$ ) while the green points $R_{\mathrm{DC}}$ are measured (with the proposed OVMC and a multimeter to monitor its accuracy) in DC operation. The DC current $I_{\mathrm{DC}}=I_{\mathrm{pk}} /(\sqrt{3} \sqrt{2})=8 \mathrm{~A}$ is selected to ensure that approximately the same losses occur in the DUT in DC operation as in TCM operation (legitimately neglecting the soft-switching losses [15]), such that $T_{\mathrm{j}, \mathrm{DC}} \approx T_{\mathrm{j}, \mathrm{TCM}}$ when $T_{\mathrm{c}, \mathrm{DC}}=T_{\mathrm{c}, \mathrm{TCM}}$ is measured. All the circuit parameters are maintained the same in both the experiments, in particular matching the value recommended in the datasheet. For Specimen A, $R_{\mathrm{TCM}}$ (cf. also Fig. 11(c)) and $R_{\mathrm{DC}}$ (Fig. 14(a)) are slightly higher than $R_{\text {data }}$ and $R_{\mathrm{DPT}}$ (cf. Fig. 13). The reason behind it is the difference in $T_{\mathrm{j}}$ between the two sets of measurements $\left(T_{\mathrm{j}, \mathrm{DC}} \approx T_{\mathrm{j}, \mathrm{TCM}}>T_{\mathrm{j}, \mathrm{data}} \approx T_{\mathrm{j}, \mathrm{DPT}}\right)$ due to the losses continuously occurring in the DUT. As a consequence, the positive temperature coefficient of $R_{\mathrm{ds}, \text { on }}$ affects the result. More interesting to notice is that $R_{\mathrm{DC}}$ is very close, i.e. within $\pm 4 \%$ (orange confidence bar is $\pm 5 \%$ ), to $R_{\mathrm{TCM}}$ as expected, since $T_{\mathrm{j}, \mathrm{DC}} \approx T_{\mathrm{j}, \mathrm{TCM}}$ and the current dependency of $R_{\mathrm{ds}, \mathrm{on}}$ is practically negligible in this range. The discrepancy can be attributed to the accuracy of the current measurement and of the OVMC, and to slightly different operating conditions. An equivalent set of measurements is performed on Specimen B in Fig. 14(b) and identical conclusions can be drawn. Hence, the accuracy and the performance of the proposed OVMC are once more validated. Fig. 14(c) summarizes $R_{\mathrm{TCM}}$ and $R_{\mathrm{DC}}$ for Specimen $\mathrm{C}$ in TABLE II. In this case a significant
Case Temperature $-T_{\mathrm{c}}\left({ }^{\circ} \mathrm{C}\right)$

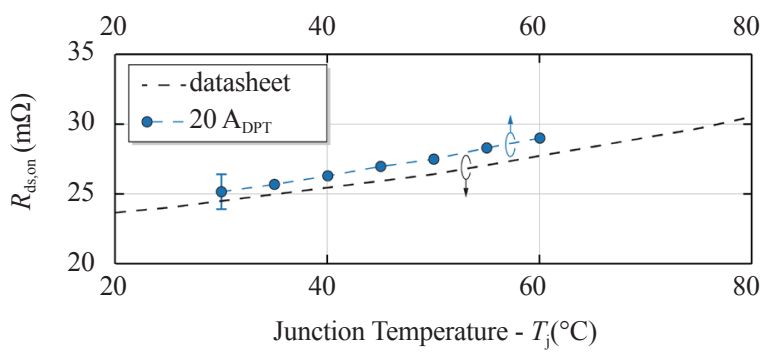

Fig. 13. Average value of $r_{\mathrm{ds}, \text { on }}$ (i.e. $\left.R_{\mathrm{DPT}}\right)$ measured with the proposed OVMC in double pulse test (DPT) operation for Specimen A in TABLE II compared with the nominal $R_{\mathrm{ds}, \text { on }}$ values reported in its datasheet (i.e. $R_{\text {data }}$ ). The measured points match the nominal values within $\pm 3 \%$.

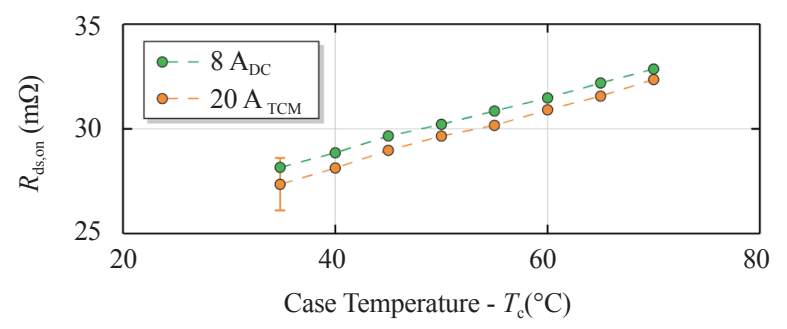

(a)

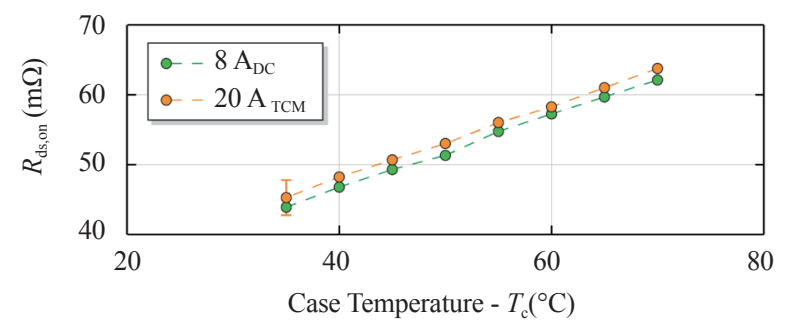

(b)

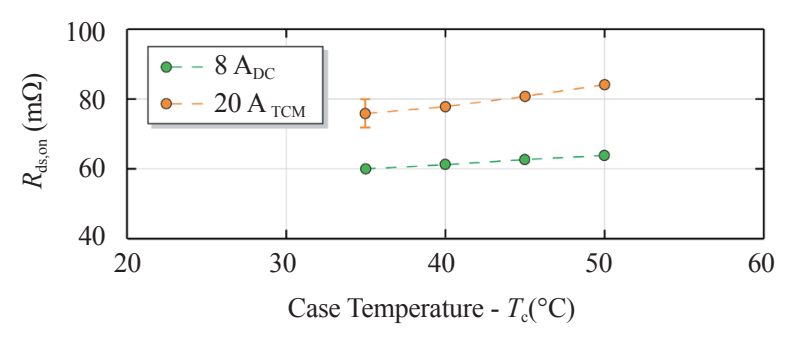

(c)

Fig. 14. Average value of $r_{\mathrm{ds}, \text { on }}$ measured with the proposed OVMC for different operating conditions of the half-bridge, for different DUTs (TABLE II) and as a function of $T_{\mathrm{c}} \cdot R_{\mathrm{TCM}}$ (orange) and $R_{\mathrm{DC}}$ (green) are similar (within $\pm 5 \%$ ) for Specimen A (a) and B (b) when the power loss conditions are matched. In the case of Specimen $\mathrm{C}$, a significant discrepancy (i.e. $\approx 50 \%$ ) between the two measurement sets is observed (c).

discrepancy (i.e. $\approx 50 \%$ ) between the two series is observed. $T_{\mathrm{j}, \mathrm{DC}} \approx T_{\mathrm{j}, \mathrm{TCM}}$ is not a true assumption anymore, since more losses are now unexpectedly occurring in the DUT in TCM operation. The discrepancy is attributed in this case to the DUT, i.e. to dynamic $R_{\mathrm{ds}, \text { on }}$ effects, which are confirmed in [7]. 


\section{Influence on Calorimetric Switching Loss Measurement}

The results illustrated in the last section can be applied to improve the accuracy of the calorimetric switching loss measurements presented in [15]. Calorimetric switching loss measurement methods, in contrast to electric switching loss measurement methods, e.g. the DPT, determine the switching losses from the measurement of thermal quantities [14], [16]. The power semiconductor under test is installed in a calorimetric test-bench, whose thermal parameters (e.g. thermal resistance and thermal capacitance) are known. While the device operates, the occurring losses generate heat and cause a variation of temperature in the test-bench. The exact amount of losses can be derived from the temperature increase.

Both conduction losses and switching losses occur simultaneously in the DUT, hence only their sum can be thermally measured. However, initially operating the DUT in the testbench at a switching frequency at which the switching losses $\left(P_{\mathrm{sw}}\right)$ can be neglected compared to the total losses $\left(P_{\mathrm{th}}\right)$, the conduction losses $\left(P_{\text {cond }}\right)$ can be accurately measured. A set of measurements performed on Specimen $C$ show an agreement between the two methods (i.e. calorimetric and OVMC) with an uncertainty in the range of 5\%, mostly attribute to the calorimetric test-bench itself [15].

The measurement method proposed in [15] to perform calorimetric switching loss measurement consists of two phases. First, accurate calorimetric measurement of the total semiconductor losses are performed $\left(P_{\mathrm{th}}\right)$. Afterwards, $P_{\text {cond }}$ are estimated and subtracted to isolate

$$
P_{\mathrm{sw}}=P_{\mathrm{th}}-P_{\mathrm{cond}} .
$$

It results immediately clear that the accuracy of the measured $P_{\mathrm{sw}}\left(\sigma P_{\mathrm{sw}}\right)$ is influenced both from the accuracy of the calorimetric measurement itself $\left(\sigma P_{\text {th }}\right)$ and of the $P_{\text {cond }}$ estimation $\left(\sigma P_{\text {cond }}\right)$. If $\sigma P_{\text {th }}=0$ is assumed for the purpose of this analysis, $\sigma P_{\mathrm{sw}}$ can be expressed, applying the propagation of uncertainty rule, as

$$
\sigma_{\% P_{\mathrm{sw}}}=-\frac{P_{\mathrm{cond}}}{P_{\mathrm{sw}}} \sigma_{\% P_{\mathrm{cond}}} .
$$

The integration of the proposed OVMC in this measurement setup aims to improve $\sigma P_{\text {cond }}$.

In [15], $R_{\mathrm{ds}, \text { on }}$ is measured in DC operation as a function of $P_{\text {th }}$, similarly to $R_{\mathrm{DC}}$ in Fig. 14(a)-(c). Afterwards an opportune (i.e. for the same $P_{\mathrm{th}}$ conditions) value of $R_{\mathrm{ds}, \mathrm{on}}$ is considered to calculate and subtract $P_{\text {cond }}$ from $P_{\text {th }}$ in TCM operation. The proposed OVMC, enabling online measurement of $R_{\mathrm{TCM}}$, has the potential to minimize $\sigma P_{\text {cond }}$, but confirmed as well the validity of the approach developed in [15] against, for example, the usage of $R_{\text {data }}$. At least for Specimen A and B, in fact, $R_{\mathrm{TCM}}$ and $R_{\mathrm{DC}}$ (orange and green points in Fig. 14(a)(b)) coincide with good approximation (within $\pm 5 \%$ ). Hence, the results shown in [15] are correct under this aspect. Differently, the discrepancy observed for Specimen C (cf. Fig.

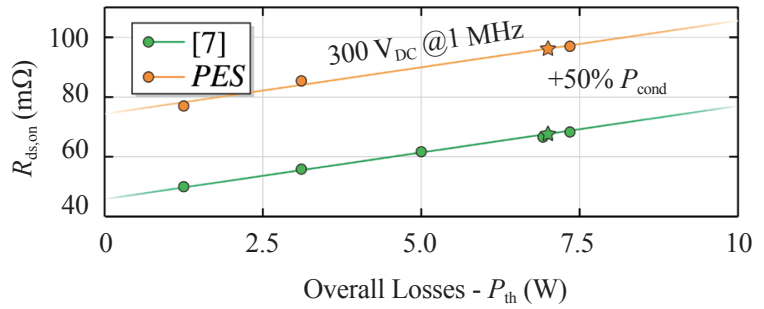

(a)

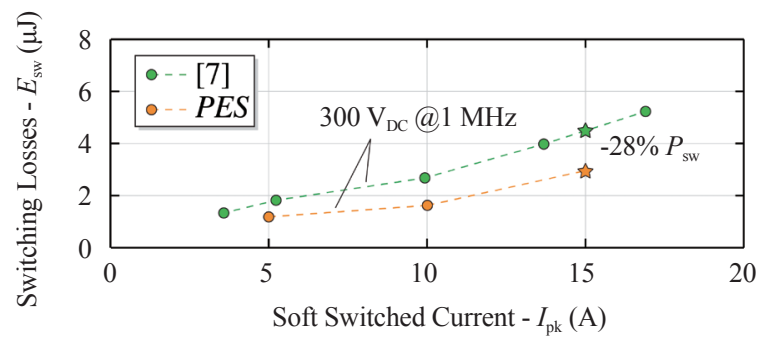

(b)

Fig. 15. Comparison between $R_{\mathrm{TCM}}$ (orange) and $R_{\mathrm{DC}}$ (green) as function of $P_{\text {th }}$ (a). The online measurement of conduction losses enabled by the proposed OVMC, more representative of the real operating conditions, results in higher (i.e. $\approx 50 \%$ ) $P_{\text {cond. }}$. The mismatch results in lower estimated $P_{\mathrm{sw}}$ (i.e. $\approx-28 \%$ ) according to the calculation reported in TABLE III (b).

TABLE III

Derivation of the Error on the Switching Losses Caused by the Inaccura-

\begin{tabular}{|c|c|c|c|}
\hline Parameter & {$[15]$} & $\begin{array}{l}\text { proposed } \\
\text { OVMC }\end{array}$ & Note \\
\hline$I_{\mathrm{pk}}(\mathrm{A})$ & 15 & & Fig. 15(b) \\
\hline$P_{\text {th }}(\mathrm{W})$ & 6.90 & 6.90 & Fig. 15(a) \\
\hline$R_{\mathrm{ds}, \text { on }}(\mathrm{m} \Omega)$ & 65 & 95 & Fig. 15(a) \\
\hline$P_{\text {cond }}(\mathrm{W})$ & 2.40 & 3.60 & $\begin{array}{l}P_{\mathrm{sw}}=R_{\mathrm{ds}, \text { on }} I_{\mathrm{rms}}^{2}, \\
I_{\mathrm{rms}}=I_{\mathrm{pk}} /(\sqrt{3} \sqrt{2})\end{array}$ \\
\hline$P_{\mathrm{sw}}(\mathrm{W})$ & 4.50 & 3.30 & $\begin{array}{l}P_{\mathrm{sw}}=f_{\mathrm{sw}} E_{\mathrm{sw}}, \\
(17) \text { and Fig. } 15(\mathrm{~b})\end{array}$ \\
\hline$\sigma \% P_{\text {cond }}$ & $50 \%$ & & $\mid \frac{P_{\text {cond },[7]}-P_{\text {cond, OVMC }}}{P_{\text {cond },[7]}}$ \\
\hline$\sigma \% P_{\mathrm{sw}}$ & $-28 \%$ & & $\begin{array}{l}\text { and Fig. 15(a) } \\
\text { (18) and Fig. 15(b) }\end{array}$ \\
\hline
\end{tabular}
Cy ON the Conduction Loss Estimation

14(c)) would introduce a significant error if the [15]-approach would be blindly adopted. Fig. 15(a) compares the results of the two conduction loss measurement methods (i.e. $R_{\mathrm{TCM}}$ and $R_{\mathrm{DC}}$ ) for one of the DUTs analyzed in [15], similar to Specimen C. In this case, the two approaches give significantly different results and the importance of OVMCs for fast switching power semiconductors is again remarked. The accurate measurement of $R_{\mathrm{TCM}}$ at $1 \mathrm{MHz}$ is the final achievement of this work, only enabled from the sophisticated design and calibration procedures described along this paper. $R_{\mathrm{TCM}}$ is up to $50 \%$ higher than $R_{\mathrm{DC}}$, i.e. $P_{\text {cond }}$ is $50 \%$ higher than previously estimated. According to (18), $P_{\mathrm{sw}}$ results up to $28 \%$ lower (see Fig. 15(b)). The physical cause behind the underlying loss mechanism is still under investigation [29]. 
The calculations for the case of $I_{\mathrm{pk}}=15 \mathrm{~A}$ are reported in TABLE III as an example.

\section{CONCLUSION}

An on-state voltage measurement circuit (OVMC) for fast switching power semiconductors is presented and fully characterized in this paper. The correction of the offset voltage present in typical OVMCs, the small input parasitic capacitance, the usage of a $50 \Omega$ output stage, the high-bandwidth conditioning circuitry, the integrated generation of the supply voltages and the thoughtful design and calibration process are key features and/or main improvements of the proposed approach when compared with state-of-the-art solutions.

The operating principle of this OVMC is described and detailed design guidelines are given. Furthermore, accurate DC and high-frequency calibration measurements are performed. Several challenges, e.g. measurement distortion due to the DUT parasitic inductance and noise amplification at low DUT current values, arise when the proposed OVMC is integrated in the measurement setup of interest considering fast switching power semiconductors. However, implementing the addressed precautions, the achieved accuracy $(<$ $\pm 2 \%)$, bandwidth $(>50 \mathrm{MHz})$ and dynamic response $(<50$ ns) finally enable precise OVMs in the case of both low $R_{\mathrm{ds}, \text { on }}$ values and at high switching frequencies, e.g. in applications featuring wide bandgap semiconductors. Ultimately, OVMs are performed on several power semiconductors for different operating conditions, and the results in terms of $R_{\mathrm{ds}, \text { on }}$ are presented, underlying the advantageous practical applicability of the circuit.

In summary, the proposed OVMC generally enables onstate behavior analysis of power semiconductors (e.g. dynamic $R_{\mathrm{ds}, \text { on }}$ effect investigation) and improves the accuracy of power converters loss breakdown models and of calorimetric switching loss measurement methods. Moreover, the OVM is envisaged as fundamental feature of next generation intelligent gate drivers, including temperature and condition monitoring, as well as time-to-failure prediction circuits.

\section{APPENDIX}

Considerations for the Accurate Tuning of the Proposed On-State Voltage Measurement Circuit

The influence of a mismatch in the diode (i.e. $\mathrm{D}_{1}$ and $\mathrm{D}_{2}$ ) voltage drops $v_{\triangle \mathrm{D}}$ can be accurately characterized. In particular, if

$$
v_{\mathrm{D}_{1}} \neq v_{\mathrm{D}_{2}}=v_{\mathrm{D}_{1}} \pm v_{\Delta \mathrm{D}},
$$

then

$$
v_{\mathrm{m}} \stackrel{(7)}{=} \frac{1}{1+\beta}\left(v_{\mathrm{ds}} \mp v_{\Delta \mathrm{D}}\right) .
$$

As in the diode-based approach of Fig. 2 (b), a mismatch in the correction of $v_{\mathrm{D}_{1}}$ translates in an offset of $v_{\mathrm{AD}}$ on $v_{\mathrm{m}}$. However, Section III-B proved how, with the necessary pre- cautions, a good accuracy can be reached. An offset on $v_{\mathrm{m}}$ can results also from resistive mismatches. First, if

$$
R_{3} \neq R_{4} \quad \longrightarrow \quad \frac{R_{3}}{R_{4}}=1 \pm \delta_{\mathrm{R}}
$$

then

$$
\begin{aligned}
& v_{\mathrm{m}} \stackrel{(4)}{=} \frac{1}{1+\beta}\left(\left(2 \pm \delta_{\mathrm{R}}\right) v_{1}-\left(1 \pm \delta_{\mathrm{R}}\right) v_{2}\right) \\
& \stackrel{(6)}{=} \frac{1}{1+\beta}\left(v_{\mathrm{ds}} \mp \delta_{\mathrm{R}} v_{\mathrm{D}_{2}}\right) .
\end{aligned}
$$

Second, if

$$
\frac{R_{1 \mathrm{~b}}}{R_{1 \mathrm{a}}+R_{1 \mathrm{~b}}} \neq \frac{R_{2 \mathrm{~b}}}{R_{2 \mathrm{a}}+R_{2 \mathrm{~b}}}=\left(1 \pm \rho_{\mathrm{R}}\right) \frac{R_{1 \mathrm{~b}}}{R_{1 \mathrm{a}}+R_{1 \mathrm{~b}}}
$$

then

$$
\begin{aligned}
& v_{\mathrm{m}} \stackrel{(4)}{=} \frac{1}{1+\beta}\left(2 v_{1}-\left(1 \pm \rho_{\mathrm{R}}\right) v_{2}\right) \\
& \stackrel{(6)}{=} \frac{1}{1+\beta}\left(v_{\mathrm{ds}} \mp \rho_{\mathrm{R}} v_{2}\right) \text {. }
\end{aligned}
$$

These information are relevant for the calibration of the proposed OVMC, e.g. understanding the causes of inaccuracy from the error trends. In particular, while $v_{\Delta \mathrm{D}}$ in (19) and $\delta_{\mathrm{R}} v_{\mathrm{D}_{2}}$ in (20) are practically constant error terms, $\rho_{\mathrm{R}} v_{2}$ in (21) is proportional to the variable measured voltage. Moreover, it results clear that precise resistors should be used for $R_{1}-R_{4}$.

\section{AcKNOWLEDGMENT}

The author would like to thank Dominik Neumayr for his precious advices and Jon Azurza Anderson for the support provided during the experiments.

\section{REFERENCES}

[1] M. Guacci, D. Bortis, I. F. Kovačević-Badstübner, U. Grossner, and J. W. Kolar, "Analysis and design of a $1200 \mathrm{~V}$ all-SiC planar interconnection power module for next generation more electrical aircraft power electronic building blocks," CPSS Transactions on Power Electronics and Applications, vol. 2, no. 4, pp. 320-330, 2017.

[2] D. Bortis, O. Knecht, D. Neumayr, and J. W. Kolar, "Comprehensive evaluation of GaN GIT in low- and high-frequency bridge leg applications," in Proc. of the IEEE 8th International Power Electronics and Motion Control Conference (IPEMC-ECCE Asia 2016), Hefei, China, 2016, pp. 21-30.

[3] D. Bortis, D. Neumayr, and J. W. Kolar, " $\eta \rho$-pareto optimization and comparative evaluation of inverter concepts considered for the GOOGLE little box challenge," in Proc. of the IEEE 17th Workshop on Control and Modeling for Power Electronics (COMPEL 2016), Trondheim, Norway, 2016, pp.1-5.

[4] T. Foulkes, T. Modeer, and R. C. N. Pilawa-Podgurski, "Developing a standardized method for measuring and quantifying dynamic on-state resistance via a survey of low voltage GaN HEMTs," in Proc. of the IEEE Applied Power Electronics Conference and Exposition (APEC 2018), San Antonio, TX, USA, 2018.

[5] "Measuring RDS(on) with high-definition oscilloscopes," Rhode \& Schwarz, 2018. 
[6] N. Badawi, O. Hilt, E. Bahat-Treidel, J. Böcker, J. Würfl, and S. Dieckerhoff, "Investigation of the dynamic on-state resistance of $600 \mathrm{~V}$ normally-off and normally-on GaN HEMTs," IEEE Transaction on Industry Application, vol. 52, no. 6, pp. 4955-4964, 2016.

[7] R. Li, X. Wu, G. Xie, and K. Sheng, "Dynamic on-state resistance evaluation of GaN Devices under hard and soft switching conditions," in Proc. of the IEEE Applied Power Electronics Conference and Exposition (APEC 2018), San Antonio, TX, USA, 2018, pp. 898-903.

[8] E. F. de Oliveira, C. Noeding, and P. Zacharias, "Impact of dynamic on-resistance of high voltage GaN switches on the overall conduction losses," in Proc. of the International Exhibition and Conference for Power Electronics, Intelligent Motion, Renewable Energy and Energy Management (PCIM Europe 2017), Nuremberg, Germany, 2017.

[9] Y. Cai, A. J. Forsyth, and R. Todd, "Impact of GaN HEMT dynamic on-state resistance on converter performance," in Proc. of the IEEE Applied Power Electronics Conference and Exposition (APEC 2017), Tampa, FL, USA, 2017, pp. 1689-1694.

[10] A. Griffo, J. Wang, K. Colombage, and T. Kamel, "Real-time measurement of temperature sensitive electrical parameters in $\mathrm{SiC}$ power MOSFETs," IEEE Transaction on Industrial Electronics, vol. 65, no. 3, pp. 2663-2671, 2017.

[11] M. A. Eleffendi and C. M. Johnson, "In-service diagnostics for wirebond lift-off and solder fatigue of power semiconductor packages," IEEE Transaction on Power Electronics, vol. 32, no. 9, pp. 7187 7198, 2017.

[12] S. Beczkowski, P. Ghimire, A. Ruiz de Vega, S. Munk-Nielsen, B. Rannestad, and P. Thøgersen, "Online vce measurement method for wear-out monitoring of high power IGBT modules," in Proc. of the 15th European Conference on Power Electronics and Applications (EPE 2013), Lille, France, 2013, pp. 1-7.

[13] M. Denk and M. -M. Bakran, "IGBT gate driver with accurate measurement of junction temperature and inverter output current," in Proc. of the International Exhibition and Conference for Power Electronics, Intelligent Motion, Renewable Energy and Energy Management (PCIM Europe 2017), Nuremberg, Germany, 2017.

[14] D. Rothmund, D. Bortis, and J. W. Kolar, "Accurate transient calorimetric measurement of soft-switching losses of $10 \mathrm{kV} \mathrm{SiC} \mathrm{MOSFETs}$ and diodes," IEEE Transaction on Power Electronics, vol. 33, no. 6 , pp. 5240-5250, 2016.

[15] D. Neumayr, M. Guacci, D. Bortis, and J. W. Kolar, "New calorimetric power transistor soft-switching loss measurement based on accurate temperature rise monitoring," in Proc. of the IEEE 29th International Symposium on Power Semiconductor Devices and ICS (ISPSD 2017), Sapporo, Japan, 2017, pp. 447-450.

[16] J. Azurza A, C. Gammeter, L. Schrittwieser, and J. W. Kolar, "Accurate calorimetric switching loss measurement for $900 \mathrm{~V} 10 \mathrm{~m} \mathrm{SiC}$ MOSFETs," IEEE Transaction on Power Electronics, vol. 32, no. 12, pp. 8963-8968, 2017.

[17] B. Carsten, "Clipping pre-amplifier for accurate scope measurement of high voltage switching transistor and diode conduction voltages," in Proc. of the 31st International Power Conversion Electronics Conference and Exhibit, 1995.

[18] K. Kaiser, "Untersuchung der verluste von pulswechselrichterstrukturen mit spannungszwischenkreis und phasenstromregelung," Ph.D. dissertation, Technische Universität Wien, 1987.

[19] N. Badawi and S. Dieckerhoff, "A new method for dynamic ron extraction of GaN Power HEMTs," in Proc. of the International Exhibition and Conference for Power Electronics, Intelligent Motion, Renewable Energy and Energy Management (PCIM Europe 2015), Nuremberg, Germany, 2015, pp.1-6.

[20] R. Gelagaev, P. Jacqmaer, and J. Driesen, "A fast voltage clamp circuit for the accurate measurement of the dynamic on-resistance of power transistors," IEEE Transaction on Industrial Electronics, vol. 62, no. 2 , pp. 1241-1250, 2015.

[21] S. Dusmez and B. Akin, "An accelerated thermal aging platform to monitor fault precursor on-state resistance," in Proc. of the IEEE International Electric Machines and Drives Conference (IEMDC 2015), Lille, France, 2015, pp. 1352-1358.

[22] P. Ghimire, A. Ruiz de Vega, S. Beczkowski, B. Rannestad, S. MunkNielsen, and P. Thøgersen, "Improving power converter reliability: online monitoring of high-power IGBT modules," IEEE Industrial
Electronics Magazine, vol. 8, no. 3, pp. 40-50, 2014.

[23] The Clipper - Springburo GmbH. (2017, November). [Online]. Available: www.springburo.weebly.com.

[24] CS1110 - Cleverscope Ltd. (2017, November). [Online]. Available: www.cleverscope.com/products/CS1110.

[25] IR25750LPBF, International Rectifier, 2015.

[26] STL2N80K5, STMicroelectronics NV, 09 2015, Rev. 3.

[27] C3D1P7060Q, Cree Inc., 10 2015, Rev. F.

[28] ADA4817-1 ADA4817-2, Analog Devices Inc., 2017, Rev. D.

[29] G. Deboy, O. Haeberlen, and M. Treu, "Perspective of loss mechanisms for silicon and wide band-gap power devices," CPSS Transactions on Power Electronics and Applications, vol. 2, no. 2, pp. 89-100, 2017.

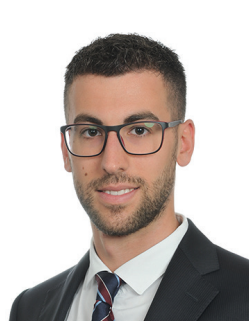

Mattia Guacci studied Electronic Engineering at the University of Udine, Italy where in July 2013 and in October 2015 he received his B.Sc. summa cum laude and his M.Sc. summa cum laude, respectively. In 2014 he was with Metasystems $\mathrm{SpA}$ in Reggio nell'Emilia, Italy working on EV onboard battery chargers. In November 2015 he joined the Power Electronic Systems Laboratory (PES) at ETH Zurich as a scientific assistant investigating innovative inverter topologies. In September 2016 he started his Ph.D. at PES focusing on integrated modular high efficiency and weight optimized power electronic converters for aircraft application.

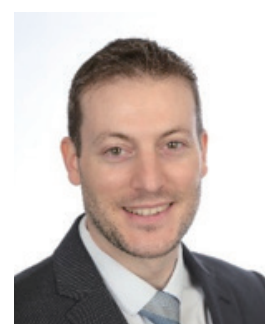

Dominik Bortis received the M.Sc. degree in electrical engineering and the Ph.D. degree from the Swiss Federal Institute of Technology (ETH) Zurich, Switzerland, in 2005 and 2008, respectively. In May 2005, he joined the Power Electronic Systems Laboratory (PES), ETH Zurich, as a Ph.D. student. From 2008 to 2011, he has been a Postdoctoral Fellow and from 2011 to 2016 a Research Associate with PES, co-supervising Ph.D. students and leading industry research projects. Since January 2016 Dr. Bortis is heading the newly established research group Advanced Mechatronic Systems at PES.

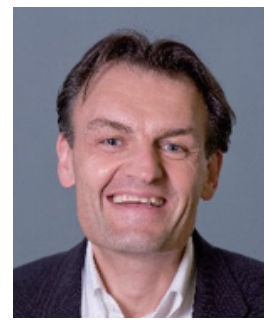

Johann W. Kolar received his M.Sc. and Ph.D degree (summa cum laude / promotio sub auspiciis praesidentis rei publicae) from the University of Technology Vienna, Austria. Since 1984, he has been working as an independent researcher and international consultant in close collaboration with the University of Technology Vienna, in the fields of power electronics, industrial electronics and high performance drives. He has proposed numerous novel PWM converter topologies, and modulation and control concepts and has supervised over $60 \mathrm{Ph} . \mathrm{D}$. students. He has published over 650 scientific papers in international journals and conference proceedings, 3 book chapters, and has filed more than 140 patents. The focus of his current research is on ultra-compact and ultraefficient $\mathrm{SiC}$ and $\mathrm{GaN}$ converter systems, wireless power transfer, solid-state transformers, power supplies on chip, and ultra-high speed and bearingless motors. Dr. Kolar has received 23 IEEE Transactions and Conference Prize Paper Awards, the 2014 IEEE Middlebrook Award, and two ETH Zurich Golden Owl Awards for excellence in teaching. He initiated and/or is the founder of four ETH Spin-off companies. He is a member of the steering committees of several leading international conferences in the field and has served from 2001 through 2013 as an Associate Editor of the IEEE Transactions on Power Electronics. Since 2002 he also is an Associate Editor of the Journal of Power Electronics of the Korean Institute of Power Electronics and a member of the Editorial Advisory Board of the IEEJ Transactions on Electrical and Electronic Engineering. 